\title{
A New Family of High Order Unstructured MOOD and ADER Finite Volume Schemes for Multidimensional Sys- tems of Hyperbolic Conservation Laws
}

\author{
Raphaël Loubère ${ }^{1, *}$ and Michael Dumbser ${ }^{2}$ and Steven Diot ${ }^{3}$ \\ ${ }^{1}$ CNRS and Institut de Mathématiques de Toulouse (IMT) Université Paul-Sabatier, Toulouse, \\ France. raphael. loubere@math.univ-toulouse.fr \\ 2 Department of Civil, Environmental and Mechanical Engineering, University of Trento, \\ Via Mesiano, 77 - 38123 Trento, Italy. michael.dumbser@unitn.it \\ ${ }^{3}$ Fluid Dynamics and Solid Mechanics (T-3), Los Alamos National Laboratory, NM 87545 \\ USA. diot@lanl.gov
}

\begin{abstract}
In this paper, we investigate the coupling of the Multi-dimensional Optimal Order Detection (MOOD) method and the Arbitrary high order DERivatives (ADER) approach in order to design a new high order accurate, robust and computationally efficient Finite Volume (FV) scheme dedicated to solve nonlinear systems of hyperbolic conservation laws on unstructured triangular and tetrahedral meshes in two and three space dimensions, respectively. The Multi-dimensional Optimal Order Detection (MOOD) method for 2D and 3D geometries has been introduced in a recent series of papers for mixed unstructured meshes. It is an arbitrary high-order accurate Finite Volume scheme in space, using polynomial reconstructions with a posteriori detection and polynomial degree decrementing processes to deal with shock waves and other discontinuities. In the following work, the time discretization is performed with an elegant and efficient one-step ADER procedure. Doing so, we retain the good properties of the MOOD scheme, that is to say the optimal high-order of accuracy is reached on smooth solutions, while spurious oscillations near singularities are prevented. The ADER technique permits not only to reduce the cost of the overall scheme as shown on a set of numerical tests in $2 \mathrm{D}$ and $3 \mathrm{D}$, but it also increases the stability of the overall scheme. A systematic comparison between classical unstructured ADER-WENO schemes and the new ADER-MOOD approach has been carried out for high-order schemes in space and time in terms of cost, robustness, accuracy and efficiency. The main finding of this paper is that the combination of ADER with MOOD generally outperforms the one of ADER and WENO either because at given accuracy MOOD is less expensive (memory and/or CPU time), or because it is more accurate for a given grid resolution. A large suite of classical numerical test problems has been solved on unstructured meshes for three challenging multi-dimensional systems of conservation laws: the Euler equations of compressible gas dynamics, the classical equations of ideal magneto-Hydrodynamics (MHD) and finally the relativistic MHD equations (RMHD), which constitutes a particularly challenging nonlinear system of hyperbolic partial differential equation. All tests are run on genuinely unstructured grids composed of simplex elements.
\end{abstract}

AMS subject classifications: 52B10, 65D18, 68U05, 68U07, 65Y05, 65Y20, $65 \mathrm{Z} 05$

Key words: Finite Volume, high-order, conservation law, polynomial reconstruction, ADER, MOOD, hyperbolic PDE, unstructured meshes, finite volume, one-step time discretization, local continuous space-time Galerkin method, WENO, Euler equations, MHD equations, relativistic MHD equations.

\footnotetext{
${ }^{*}$ Corresponding author.
} 


\section{Introduction and context}

This paper deals with the development of a new family of arbitrary high order accurate finite volume schemes in space and time for the solution of nonlinear systems of hyperbolic partial differential equations, as for instance the Euler equations of compressible gas dynamics, or the ideal classical and relativistic magneto-hydrodynamics system (MHD/RMHD). More precisely, we propose to couple the recently developed a posteriori "Multi-dimensional Optimal Order Detection" (MOOD) concept $[18,29,30]$ with the "Arbitrary high order DERivatives" (ADER) scheme, that allows us to reach arbitrary order of accuracy in space and time in one single step. The resulting method will be denoted by ADER-MOOD in the following.

By 'higher order' we strictly consider better than second order accurate methods, whose effective numerical order of accuracy is optimal for smooth solutions. More importantly, such numerical methods must be efficient on general unstructured meshes in multiple space dimensions. Several of such methods are available under the key names Discontinuous Galerkin (DG) methods [19, 21-24,40], ENO, WENO and HWENO methods (Weighted/Hermite Essentially Non Oscillatory) $[1,4,9,16,50,52,55,57,58,68-70,76,85,92]$, ADER schemes $[2,8,9,37-39,59,74,75,83,84,86,88,91]$, $P_{N} P_{M}$ schemes [32,34,62,63], etc.

By efficient we understand that such methods can be implemented with acceptable effort and must be numerically validated on a test suite as exhaustive as possible; they can run on computers of reasonable size, and they must be robust, stable and accurate. This set of properties seems to be a general agreement amongst developers of numerical methods for conservation laws.

To avoid Gibbs phenomenon that occurs when shock waves or steep gradients are present, any better than first order accurate scheme must add some sort of extra dissipation usually manifested by a non linearity in the scheme. This is a direct consequence of the Godunov theorem, which states that there exist no better than first order accurate linear schemes for hyperbolic transport equations. The nonlinearity is usually introduced into the scheme under the form of a so-called 'limiter'. Many different sorts have been designed: slope limiters for piecewise linear reconstructions (Monotone Upstream Schemes for Conservation Laws (MUSCL) [60,93,94]), flux limiter techniques (Flux Corrected Transport FCT methods $[14,15])$, or higher order methods that choose the least oscillatory piecewise polynomial reconstruction (ENO, WENO, HWENO methods) on different candidate stencils, or the artificial viscosity approach commonly used for finite element methods or Lagrangian schemes, for instance.

Most of these limitation techniques are effective in special cases but none of them is generic enough and/or efficient enough. Such limitation techniques are a priori in the sense that from a discrete solution at discrete time $t^{n}$ the limiter must deduce the location of its action and its intensity to ensure that the solution at $t^{n+1}$ is valid. Usually this task is achieved, but with too much intensity leading to a solution which could be less limited, and could therefore be more accurate. Moreover, some limiting techniques sometimes rely on parameters that have to be fixed, and whose dependencies on the test problem are not always clear. Note that some of these techniques are still on-going subject of research in their respective fields. This indicates that no agreement has been reached yet on how and where the extra dissipation should be properly introduced into a high order numerical method for nonlinear systems of conservation laws in multiple space dimensions.

In this work we focus on finite volume schemes on fixed unstructured meshes working with piecewise constant data at the beginning of each timestep. The main representatives of this family of numerical methods are the second order MUSCL approach and the family of (W)ENO schemes. Both techniques share some common features, namely 
- They are finite volume methods, i.e. the data are given as cell averages of conservative variables.

- A numerical flux, e.g. a Riemann solver, is employed for flux computation at cell interfaces.

- High-order in space is achieved based on polynomial reconstructions. One piecewise linear polynomial per variable per cell is reconstructed for MUSCL whereas higher order polynomials are recovered from the cell averages for ENO type methods. These reconstructions feed the numerical flux with high-order reconstructed boundary states at cell interfaces.

- All classical higher order finite volume methods need to insert some sort of a priori limitation of the reconstructions to avoid Gibbs phenomenon. A slope limiter is typically used for MUSCL whereas (W)ENO methods use several polynomial reconstructions on different stencils to construct an essentially non-oscillatory reconstruction.

- Time integration can be either done using the method of lines, based on a TVD Runge-Kutta scheme [48], or using a one-step approach, like the ADER scheme.

The recently developed Multi-dimensional Optimal Order Detection (MOOD) concept has proposed an a posteriori approach to the problem of limitation. Indeed the mojo of this paradigm is to run a spatially unlimited high-order finite volume scheme to get a candidate solution. Then the validity of the candidate solution is tested against a set of predefined admissibility criteria. Some cells are marked as 'acceptable' and are therefore valid. Some others may be locally marked as 'problematic' if they do not pass the detection process. These cells and their neighbors are consequently locally recomputed using polynomial reconstructions of a lower degree. Thus, after decrementing the polynomial degree and local recomputing of the solution, a new candidate solution is obtained. That solution is again tested for validity and the decrementing procedure re-applies, if necessary.

Such degree decrementing can occur several times within one time step for the same cell, but it will always halt after a finite number of steps: either the cell is valid for a polynomial degree greater than 0 , or the degree zero is reached. In the latter case, the worst case scenario, the cell is updated with the robust and stable first order accurate Godunov finite volume scheme, which is supposed to always produce valid solutions. This a posteriori check and decrementing iteration loop is called the 'MOOD loop'. We refer the reader to $[18,29,30]$ for more details.

Originally in $[18,29,30]$ the third order TVD Runge-Kutta method in time was used (RK3), together with a suitable timestep restriction in order to reach very high order of accuracy in time. This scheme was not uniformly high order accurate in space and time and the RK3 time discretization was also rather expensive due to the multiplication of solution solves required by the Runge-Kutta substages within one time step.

In recent years, one-step ADER (Arbitrary high order DERivatives) time stepping methods have emerged as competitors to the RK time stepping methods, see e.g. [64, 89], [83], [90], [86], [36], [34], $[8,9],[17]$. The methods were initially formulated by Toro, Millington and Nejad in [64] and numerical implementations were presented in Titarev and Toro [83]. While the original ADER approach of Titarev and Toro approximately solves the generalized Riemann problem (GRP) (van Leer [94], BenArtzi and Falcovitz [12]) at zone boundaries, the most recent ADER methods $[8,9,34,36]$ evolve the spatially high order accurate reconstruction polynomial locally in time using a weak integral formulation of the conservation law in space-time, and, as such, obtain a space-time accurate representation of the solution within a cell. At each zone boundary, classical Riemann problems are then solved with the higher order boundary extrapolated values of the space-time polynomials. An efficient quadrature-free approach has been proposed in [38]. This most recent version of the ADER schemes is more similar 
to the original ENO scheme proposed by Harten et al. [49], since it first evolves the data in each element by solving a local Cauchy problem in the small, i.e. without accounting for the neighbor cells, and then solves the interactions at the zone boundaries. The scheme therefore follows a reconstruct evolve - update strategy.

Existing high order ADER-WENO finite volume schemes are attractive because they are uniformly high order accurate one-step methods in space and time for any high order polynomial degree. However, an important practical limitation is introduced by the rather heavy and complex WENO reconstruction methodology: heavy, because several polynomial reconstructions (one on each stencil) are required for each variable in each cell; complex, because WENO should employ characteristics variables, which can be rather expensive to calculate or may even not be available in closed form for certain hyperbolic systems. On the opposite, MOOD schemes propose an efficient nonlinear a posteriori limiting based on a set of predefined admissibility criteria for any high order polynomial degree of the spatial reconstruction operator, but were limited so far to formally third order of accuracy in time due to the use of classical TVD Runge-Kutta time stepping [48]. As a consequence, the combination of the spatially high order accurate MOOD framework with the latest generation of high order one-step ADER schemes seems to be reasonable and may lead to accurate, efficient and robust schemes for multidimensional systems of hyperbolic conservation laws. The purpose of this work can therefore be summarized as follows:

1. Present how MOOD and ADER schemes can be efficiently coupled and how they naturally lead to an efficient high-order accurate one-step method in space and time;

2. Numerically validate this ADER-MOOD scheme by

- showing the effective high-order of accuracy in space and time on smooth solutions;

- comparing this method with existing methods of the same kind. We choose the classical ADER-WENO scheme in characteristic variables for comparison and we provide several diagnostics such as accuracy measures, CPU time, memory consumption, visualization versus exact or reference solutions, etc. to provide a fair comparison.

3. Present how the MOOD paradigm can be adapted to other systems of conservation laws such as the MHD and relativistic MHD system. As such we emphasize the flexibility of the approach. Numerical validation on known problems are also provided along with comparisons with ADERWENO results.

4. Underline by the results obtained in this work that the MOOD paradigm is non invasive to an already existing code. The MOOD loop has been implemented into the existing 3D MPI parallel $\mathbb{P}_{N} \mathbb{P}_{M}$ framework for general hyperbolic systems of conservation laws presented in [34]. Very few efforts were necessary to generate a working high order ADER-MOOD method, the results of which are provided in this paper.

The remaining part of this paper is organized as follows. In section 2 we present the unlimited arbitrary high-order ADER finite volume scheme. Then, in section 3, we present the MOOD paradigm. In particular, we precise which low-order scheme is considered, which cascade of schemes is used and, the cornerstone, which numerical properties and admissibility criteria the candidate solution must fulfill to be locally considered as acceptable. Then in section 3.1 three systems of conservation laws are considered, namely the hydrodynamics (HD) Euler equations for compressible fluids, the equations of magneto-hydrodynamics (MHD) and finally the relativistic magneto-hydrodynamics equations 
(RMHD). In all cases the physical admissibility criteria are derived from the underlying physics.

Then, a large section on numerical test problems provides the reader with results for numerous test cases for the Euler system (isentropic vortex, double Mach reflection problem, planar shock tube problems, spherical 3D explosion problem), the MHD system (Orszag-Tang vortex, MHD rotor problem) and the RMHD system (large amplitude Alfvén wave, relativistic blast wave and rotor problem). These problems are run in 2D or 3D on unstructured meshes and all are used to compare the new ADER-MOOD approach with the classical ADER-WENO method. The last section concludes this paper by presenting future perspectives of research.

\section{Unlimited ADER Finite Volume Schemes}

Let us consider the following general form of a nonlinear hyperbolic system of conservation laws in multiple space dimensions

$$
\frac{\partial \mathbf{Q}}{\partial t}+\nabla \cdot \mathbf{F}(\mathbf{Q})=0, \quad \mathbf{x} \in \Omega \subset \mathbb{R}^{d}, \quad t \in \mathbb{R}_{0}^{+},
$$

with appropriate initial and boundary conditions

$$
\mathbf{Q}(\mathbf{x}, 0)=\mathbf{Q}_{0}(\mathbf{x}), \quad \forall \mathbf{x} \in \Omega, \quad \mathbf{Q}(\mathbf{x}, t)=\mathbf{Q}_{B}(\mathbf{x}, t) \quad \forall \mathbf{x} \in \partial \Omega, \quad t \in \mathbb{R}_{0}^{+} .
$$

Here, $\mathbf{Q} \in \Omega_{Q} \subset \mathbb{R}^{\nu}$ is the vector of $\nu$ conserved quantities, or the so-called state vector and $\mathbf{F}(\mathbf{Q})=$ $(\mathbf{f}, \mathbf{g})$ is a nonlinear flux tensor that depends on the state $\mathbf{Q} . \Omega$ denotes the computational domain in $d$ space dimensions and $\Omega_{Q}$ is the space of physically admissible states or phase-space. In this paper we solve this system of equations by applying the high order accurate $\mathbb{P}_{N} \mathbb{P}_{M}$ method recently proposed by [33] maintaining at the same time better than second order of accuracy in space and time. We focus in this work on the finite volume family of schemes referred to as $\mathbb{P}_{0} \mathbb{P}_{M}$ schemes. The numerical method is formulated as one-step local predictor global corrector method. The predictor is based on an element-local weak solution of equation (2.1), where inside each element the governing PDE (2.1) is solved in the small (see [49]) by means of a locally implicit space-time discontinuous Galerkin scheme. This leads to an algebraic system of non-linear equations that must be solved individually for each element. The globally explicit update in time, on the other hand, is obtained by a standard finite volume approach. In the following we only summarize the main steps, while for more details the reader is referred to $[8,32,33,35,36,43,46,53]$.

\subsection{The $\mathbb{P}_{0} \mathbb{P}_{M}$ Reconstruction Operator on Unstructured Meshes}

The main ingredient of the proposed numerical method to reach high order of accuracy in space is the $P_{N} P_{M}$ reconstruction operator on unstructured meshes first introduced in [33]. We focus on the special case of finite volume schemes $(N=0)$ proposed in $[37,38]$ and further denoted by $\mathbb{P}_{0} \mathbb{P}_{M}$ or simply $\mathbb{P}_{M}$. For the details, we refer to the above mentioned publications and give only a short review in this section.

The computational domain $\Omega$ is discretized by conforming elements $T_{i}$, where the index $i$ ranges from 1 to the total number of elements $N_{E}$. The elements are chosen to be simplex elements, hence triangles in $2 \mathrm{D}$ and tetrahedrons in $3 \mathrm{D}$. The union of all elements is called the triangulation or 
tetrahedrization of the domain, respectively,

$$
\mathcal{T}_{\Omega}=\bigcup_{i=1}^{N_{E}} T_{i} .
$$

At the beginning of a time-step, the numerical solution of equation (2.1) for the state vector $\mathbf{Q}$, denoted by $\mathbf{u}_{h}\left(\mathbf{x}, t^{n}\right)$, is represented by piecewise constant cell averages

$$
\mathbf{u}_{i}^{n}=\frac{1}{\left|T_{i}\right|} \int_{T_{i}} \mathbf{Q}\left(\mathbf{x}, t^{n}\right) d \mathbf{x}, \quad \text { with } \quad\left|T_{i}\right|=\int_{T_{i}} d \mathbf{x} .
$$

The piecewise constant solution $\mathbf{u}_{h}$ is therefore given in terms of the cell averages by

$$
\mathbf{u}_{h}\left(\mathbf{x}, t^{n}\right)=\mathbf{u}_{i}^{n} \quad \text { if } \quad \mathbf{x} \in T_{i} .
$$

From the piecewise constant data representation $\mathbf{u}_{h}\left(\mathbf{x}, t^{n}\right) \in \mathcal{V}_{h}$, we then reconstruct piecewise polynomials $\mathbf{w}_{h}\left(\mathbf{x}, t^{n}\right)$ of maximum degree $M \geq 0$ from the space $\mathcal{W}_{h}$, spanned by the basis functions $\Psi_{l}=\Psi_{l}(\mathbf{x})$ :

$$
\mathbf{w}_{h}\left(\mathbf{x}, t^{n}\right)=\sum_{l} \Psi_{l}(\mathbf{x}) \hat{\mathbf{w}}_{l}^{n}
$$

As stated in [33], the $\Psi_{l}$ form an orthogonal basis on the simplex, for example the Dubiner basis [20,31]. The reconstruction on element $T_{i}$ then requires a so-called reconstruction stencil, i.e. an appropriate neighborhood of element $T_{i}$ that we choose as

$$
\mathcal{S}_{i}=\bigcup_{k=1}^{n_{e}} T_{j(k)},
$$

containing a total number of $n_{e}$ elements. Here $1 \leq k \leq n_{e}$ is a local index, counting the elements in the stencil, and $j=j(k)$ is the mapping from the local index $k$ to the global indexation of the elements in $\mathcal{T}_{\Omega}$. For ease of notation, we omit in the following the dependency on $k$ for $j(k)$ and only write $j$. The stencil $\mathcal{S}_{i}$ can be constructed automatically by recursively adding neighbors and neighbors of neighbors of element $T_{i}$ to a list, which yields the stencil $\mathcal{S}_{i}$. For the unlimited version of the scheme presented in this work, only one central reconstruction stencil is necessary, in contrast to the WENO reconstruction, which needs several reconstruction stencils, see $[37,38]$.

In the present paper the three operators

$$
\begin{gathered}
\langle f, g\rangle_{T_{i}}=\int_{t^{n}}^{t^{n+1}} \int_{T_{i}}(f(\mathbf{x}, t) \cdot g(\mathbf{x}, t)) d \mathbf{x} d t, \\
{[f, g]_{T_{i}}^{t}=\int_{T_{i}}(f(\mathbf{x}, t) \cdot g(\mathbf{x}, t)) d \mathbf{x},} \\
\{f, g\}_{\partial T_{i}}=\int_{t^{n}}^{t^{n+1}} \int_{\partial T_{i}}(f(\mathbf{x}, t) \cdot g(\mathbf{x}, t)) d S d t,
\end{gathered}
$$

respectively denote the scalar products of two functions $f$ and $g$ over the space-time element $T_{i} \times$ $\left[t^{n} ; t^{n+1}\right]$, over the spatial element $T_{i}$, and over the space-time element boundary $\partial T_{i} \times\left[t^{n} ; t^{n+1}\right]$. The 
operators $\langle f, g\rangle$ and $[f, g]^{t}$, written without the index $T_{i}$, will denote scalar products on the spacetime reference element $T_{E} \times[0 ; 1]$ and on the spatial reference element $T_{E}$ at time $t$, respectively. The spatial reference element $Q_{E}$ is defined as the unit simplex with vertices $(0,0),(1,0),(0,1)$ in two space dimensions and vertices $(0,0,0),(1,0,0),(0,1,0)$ and $(0,0,1)$ in three space dimensions, respectively.

The reconstruction is now obtained via $L_{2}$-projection of the (unknown) piecewise polynomials $\mathbf{w}_{h}$ from the space $\mathcal{W}_{h}$ onto the space $\mathcal{V}_{h}$ on each stencil $\mathcal{S}_{i}$ as follows:

$$
\left[1, \mathbf{w}_{h}\right]_{T_{j}}^{t^{n}}=\left[1, \mathbf{u}_{h}\right]_{T_{j}}^{t^{n}}, \quad \forall T_{j} \in \mathcal{S}_{i},
$$

i.e. we require integral conservation in order to obtain the reconstruction polynomial $\mathbf{w}_{h}$.

During the reconstruction step, the polynomials $\mathbf{w}_{h}$ are continuously extended over the entire stencil $\mathcal{S}_{i}$. After reconstruction, the piecewise polynomials $\mathbf{w}_{h}$ are again restricted onto each element $T_{i}$. The number of elements in the stencils are chosen in such a way that the number of equations in equation (2.11) is larger that the number of degrees of freedom in the space $\mathcal{W}_{h}$. Therefore, equation (2.11) constitutes an overdetermined linear algebraic equation system for the coefficients of $\mathbf{w}_{h}$ and is solved using a constrained least squares technique, see $[33,37]$. In alternative, a much more elegant kernel based reconstruction can be used [2] and its extension to the general family of $\mathbb{P}_{N} \mathbb{P}_{M}$ schemes of [33] is current research in progress. The linear constraint to be imposed for the constrained least squares method stipulates that equation (2.11) is exactly satisfied for $T_{j}=T_{i}$, i.e. inside the element $T_{i}$ under consideration. The integral on the left hand side in equation (2.11) is computed using classical multidimensional Gaussian quadrature of appropriate order, see [79]. For the pure finite volume case, the unlimited $\mathbb{P}_{0} \mathbb{P}_{M}$ reconstruction used here corresponds to the reconstruction operator proposed by Barth and Frederickson in their pioneering work [10].

\subsection{The Local Space-Time Predictor}

The reconstruction polynomials $\mathbf{w}_{h}\left(\mathbf{x}, t^{n}\right)$ are now evolved in time according to a local weak formulation of the governing PDE in space-time, see $[8,33,36,43,46,53]$. We underline that the local space-time Galerkin method is only used for the construction of an element-local predictor solution of the PDE (without the influence of the neighbors), and is later inserted into a corrector that provides the appropriate coupling between neighbors.

Transformation of the PDE (2.1) into a space-time reference coordinate system $(\boldsymbol{\xi}, \tau)$ of the spacetime reference element $T_{E} \times[0 ; 1]$ with $\nabla_{\xi}=\partial \boldsymbol{\xi} / \partial \mathbf{x} \cdot \nabla$ yields

$$
\frac{\partial \mathbf{Q}}{\partial \tau}+\nabla_{\xi} \cdot \mathbf{F}^{*}(\mathbf{Q})=0
$$

with

$$
\mathbf{F}^{*}:=\Delta t(\partial \boldsymbol{\xi} / \partial \mathbf{x})^{T} \cdot \mathbf{F}(\mathbf{Q}) .
$$

Multiplication of equation (2.12) with a space-time test function $\theta_{k}=\theta_{k}(\boldsymbol{\xi}, \tau)$ from the space of piecewise space-time polynomials of degree $M$ and integration over the space-time reference control volume $T_{E} \times[0 ; 1]$ yields the following weak formulation:

$$
\left\langle\theta_{k}, \frac{\partial \mathbf{q}_{h}}{\partial \tau}\right\rangle+\left\langle\theta_{k}, \nabla_{\xi} \cdot \mathbf{F}_{h}^{*}\left(\mathbf{q}_{h}\right)\right\rangle=0
$$

For the discrete solution of equation (2.14) in space-time $\mathbf{q}_{h}$ we use the same ansatz for $\mathbf{q}_{h}$ as well as for $\mathbf{F}_{h}^{*}$, i.e.

$$
\mathbf{q}_{h}=\mathbf{q}_{h}(\boldsymbol{\xi}, \tau)=\sum_{l} \theta_{l}(\boldsymbol{\xi}, \tau) \hat{\mathbf{q}}_{l}:=\theta_{l} \hat{\mathbf{q}}_{l}
$$




$$
\mathbf{F}_{h}^{*}=\mathbf{F}_{h}^{*}(\boldsymbol{\xi}, \tau)=\sum_{l} \theta_{l}(\boldsymbol{\xi}, \tau) \hat{\mathbf{F}}_{l}^{*}:=\theta_{l} \hat{\mathbf{F}}_{l}^{*}
$$

Using a nodal basis [33], one simply has

$$
\hat{\mathbf{F}}_{l}^{*}=\mathbf{F}^{*}\left(\hat{\mathbf{q}}_{l}\right) .
$$

Integration by parts in time of the first term in (2.14) yields

$$
\left[\theta_{k}, \mathbf{q}_{h}\right]^{1}-\left[\theta_{k}, \mathbf{w}_{h}\right]^{0}-\left\langle\frac{\partial}{\partial \tau} \theta_{k}, \mathbf{q}_{h}\right\rangle+\left\langle\theta_{k}, \nabla_{\xi} \cdot \mathbf{F}_{h}^{*}\right\rangle=0
$$

where the piecewise high order polynomial reconstruction $\mathbf{w}_{h}$ is taken into account as initial condition of the Cauchy problem in the small in a weak sense by the term $\left[\theta_{k}, \mathbf{w}_{h}\right]^{0}$.

Inserting (2.15) into (2.18) one gets

$$
\left(\left[\theta_{k}, \theta_{l}\right]^{1}-\left\langle\frac{\partial}{\partial \tau} \theta_{k}, \theta_{l}\right\rangle\right) \hat{\mathbf{q}}_{l}+\left\langle\theta_{k}, \nabla_{\xi} \theta_{l}\right\rangle \cdot \mathbf{F}^{*}\left(\hat{\mathbf{q}}_{l}\right)=\left[\theta_{k}, \Psi_{l}\right]^{0} \hat{\mathbf{w}}_{l}^{n}
$$

which can be easily solved for the unknown expansion coefficients $\hat{\mathbf{q}}_{l}$ of the local space-time predictor solution by an element-local fixed-point iteration scheme, see $[33,43,53]$.

\subsection{The Fully Discrete One-Step ADER Scheme}

The fully discrete one-step ADER scheme is obtained by integrating the governing PDE over the spacetime control volume $T_{i} \times\left[t^{n} ; t^{n+1}\right]$, hence by applying the operator $\langle 1, \cdot\rangle_{T_{i}}$ to PDE (2.1). Application of Gauss theorem, introduction of a numerical flux function $\mathcal{G}_{i j} \cdot \mathbf{n}_{i j}$ and insertion of the local predictor solution $\mathbf{q}_{h}$ into the computation of the numerical fluxes at the element boundaries in normal direction $\mathbf{n}_{i j}$ leads to the following conservative, high order accurate one-step finite volume scheme:

$$
\mathbf{u}_{i}^{n+1}=\mathbf{u}_{i}^{n}-\frac{1}{\left|T_{i}\right|} \sum_{T_{j} \in \mathcal{N}_{j}} \int_{t^{n}}^{t^{n+1}} \int_{\partial T_{i j}} \mathcal{G}_{i j}\left(\mathbf{q}_{h}^{-}, \mathbf{q}_{h}^{+}\right) \cdot \mathbf{n}_{i j} d S d t .
$$

Here, $\mathbf{q}_{h}^{-}$and $\mathbf{q}_{h}^{+}$denote the boundary extrapolated data from element $T_{i}$ and its neighbor $T_{j}$ at the common interface $\partial T_{i j}$, respectively. The set of direct side neighbors of element $T_{i}$ is denoted by $\mathcal{N}_{j}$. In all test cases presented in this paper we use as numerical flux function at the element boundaries either the simple Rusanov (local Lax Friedrichs) flux [73], or the general Osher-type flux recently proposed in $[41,42]$.

\section{MOOD: an a posteriori limiter paradigm}

The MOOD (Multi-dimensional Optimal Order Detection) approach has been introduced in three recent papers $[18,29,30]$ as an original a posteriori technique to prevent the creation of Gibbs phenomenon at discontinuities and has been proved to be a competitive alternative to the well-known WENO method that is widely used in the design of higher-order schemes. In particular, the original ADER schemes rely on high order polynomial WENO reconstructions for $\mathbf{w}_{h}[35,37,38,50,52,78]$.

We recall that the WENO polynomial is the essentially non-oscillatory polynomial of degree $M$, which can be constructed from as many reconstructed polynomials as we can afford by considering 
different reconstruction stencils. For a 2D triangulation the number of stencils to be considered is 7 (1 central stencil, 3 forward stencils and 3 backward stencils). On tetrahedral meshes in 3D, 9 reconstructions per variable and per cell are used, see [37,38]. When $M$ is greater but close to 3 the extra-cost brought by these successive reconstructions is important. Beyond $M=5$, this may become the bottleneck of the method. Not only the CPU time may become large but also the memory consumption becomes excessively high, in particular for high order WENO schemes in 3D; this further decreases the performance of the whole method and may be considered as a weakness of ADER-WENO methods that a MOOD approach may mitigate.

\subsection{Basics of the MOOD paradigm}

The MOOD method $[18,29,30]$ is based on an a posteriori evaluation of the solution to determine if some sort of limitation has to be applied and where.

The technique is a posteriori in the sense that we compute a candidate solution $\mathbf{u}_{h}^{*}\left(\mathbf{x}, t^{n+1}\right)$, i.e. a potential approximation of the solution at time $t^{n+1}$, and then one determines if this candidate solution is acceptable, or not. More precisely, the detection-limitation mechanism operates in several steps. A candidate solution is first computed with the highest-order unlimited scheme (the polynomials with maximal degree $M$ ). Then a detection procedure is performed to determine the problematic cells, i.e. all cells where the approximation does not respect some given criteria. The solution is locally recomputed with a lower-order scheme (using polynomial reconstructions of lower degree). This procedure, called the MOOD loop, is repeated until each cell satisfies all detection criteria or the polynomial degree has reached the smallest possible value. In the last case, a robust scheme, as for instance a positivity preserving first order finite volume scheme, is triggered and a meaningful physical solution is provided. Throughout this paper we suppose that the first order method is robust enough to provide always a physically admissible solution, although finding such a scheme may be non-trivial for very complex nonlinear hyperbolic systems.

In this work we have replaced the WENO technique originally employed in the ADER-WENO schemes by the non-invasive and more efficient a posteriori MOOD paradigm. Moreover, while WENO needs the knowledge of characteristics variables MOOD only considers conservative variables.

When a MOOD loop embraces an already existing code we have to provide answers to the following questions:

1. What are the constraints that a candidate solution must fulfill for the code to be able to compute the next time step? In other words, given the system of equations one solves, what are the minimal requirements to be demanded to a numerical solution to ensure that the code does not prematurely terminate. Some authors refer to this issue as ensuring 'admissible states'. For example in the case of the hydrodynamics equations with a perfect gas equation of state, one must ensure that the discrete density, $\rho_{i}$, and the specific internal energy, $E_{i}-\frac{1}{2} \mathbf{v}_{i}^{2}$, are both positive to be able to further compute positive pressures and to have a well defined sound speed. The authors of $[18,29,30]$ refer to these constraints as Physical Admissibility Detection (PAD) constraints.

For the relativistic magneto-hydrodynamics system (RMHD) one must also require that the velocity remains less than the speed of light, in order to be consistent with relativistic physics. For more complex systems of equations these requirements may involve additional quantities, such as entropy [28], temperature or any other physically meaningful variables.

Note that these constraints do ensure neither accuracy nor stability of the numerical method. 
They simply avoid the unpleasant situation of code crashing. As such they are not 'negotiable' when designing a numerical scheme.

2. Which scheme is the 'bullet-proof' or 'parachute' scheme? In other words, which scheme amongst all possible schemes implemented in the code is the scheme you, as a developer, do trust. This scheme must provide a bullet-proof solution when everything else fails. For instance in $[18,29,30]$ the authors rely on the first-order finite volume scheme. In fact, any other scheme which ensures that admissible states are provided (hence if all PAD constraints are satisfied) can be used.

3. Which cascade of schemes is considered? The goal is to rank all numerical schemes from the 'bullet-proof' scheme (inaccurate but always robust) up to the 'most performing' scheme (highly accurate but less robust). For instance in $[18,29,30]$ the authors propose to use the cascade $\mathbb{P}_{M} \longrightarrow \mathbb{P}_{2} \longrightarrow \mathbb{P}_{0}$ of unlimited schemes with $M=3$ or 5 .

4. What are the constraints that a candidate solution should fulfill to be considered as acceptable? These constraints must ensure the stability of the solution, but also some more or less important features that the developer desires. For example in $[18,29,30]$ for the hydrodynamics system of equations the authors rely on the relaxed Discrete Maximum Principle (DMP) by the so-called $\mathrm{u} 2$ detection process based on local regularity of the density variable. This detection criterion ensures to obtain higher-order of accuracy for regular solutions while preventing spurious numerical oscillations in the vicinity of discontinuous profiles. However, for other systems of equations different variables may be more appropriate to be checked than the density only. In reality each practitioner has its own view on what should be a good solution, or, how a solution should look like. Let us call refer to theses constraints as Numerical Admissibility Detection (NAD) constraints.

The efficiency of the a posteriori MOOD paradigm is brought by the fact that usually few cells need a cell polynomial degree decrementing, therefore the extra-work needed to recompute a new candidate solution on problematic cells is low. Moreover, for a given degree only one polynomial reconstruction per variable and per cell is computed, which reduces not only the CPU time but also the memory consumption compared to an a priori WENO reconstruction.

The flexibility of the a posteriori MOOD paradigm is mainly due to the fact that (1) a robust preferred parachute scheme can be kept as the last and safest scheme, (2) only few modifications are usually needed within the code because the MOOD loop embraces the existing solver, (3) any constraint can be added to the list of detection criteria, should they be physics-based or numerics-based or simply the developer's whim.

In this work the MOOD loop has been implemented within an existing 3D $\mathbb{P}_{N} \mathbb{P}_{M}$ code $[36-38,82]$ for conservative and non-conservative hyperbolic systems and has been parallelized using the MPI standard. Obviously, for parallel computations with MPI some extra communications are needed in the case of MOOD, namely if a cell has to be recomputed with decremented polynomial degree. However, WENO finite volume schemes also require a substantial effort of MPI communications due to the necessary data exchange over many stencils per cell. But while WENO always requires the same amount of communications within a simulation, the MOOD framework may require dynamically more or less MPI communications according to the current numerical solution and the NAD and PAD criteria used. In this sense, reaching optimal MPI load balancing may be more complicated with the MOOD approach. 
In the present implementation, the MOOD loop simply embraces the main evolution routines of the high order ADER method and iterates to recompute those cells with invalid values, detected by the PAD and NAD admissibility criteria, see the next section for some examples. Our choice as developers was to use the following cascade: $\mathbb{P}_{M} \longrightarrow \mathbb{P}_{1}^{\text {lim }} \longrightarrow \mathbb{P}_{0}$. We have taken $M=3$, but other values of $M$ could be used as well (some test cases use $M>3$ in the section on numerical test problems). The $\mathbb{P}_{1}^{\text {lim }}$ scheme uses $\mathbb{P}_{1}$ reconstructions with Barth \& Jespersen slope limiting [11].

More precisely, the MOOD loop first computes the unlimited $\mathbb{P}_{M}$ candidate solution $u_{h}^{*}\left(\mathbf{x}, t^{n+1}\right)$, checks if any cell is problematic according to developer-given constraints (see 1. above). Then all invalid cells and their neighbors are recomputed with the $\mathbb{P}_{1}^{\text {lim }}$ scheme and re-checked for validity. Note that such a candidate solution may enjoy $\mathbb{P}_{3}$-updated cells and $\mathbb{P}_{1}^{\text {lim }}$-updated ones. Nonetheless, some cells may still be invalid because the $\mathbb{P}_{1}^{\text {lim }}$ may still compute some non-admissible states, for instance the pressure positivity for Euler equations is difficult to fulfill under a reasonable CFL condition. As a consequence, for these still invalid cells the last scheme $\mathbb{P}_{0}$ is triggered. Being the 'parachute' scheme any remaining problematic cells are properly updated, in the sense that the PAD criteria are fulfilled.

In the worst case scenario all cells in the domain are updated with the first-order $\mathbb{P}_{0}$ scheme. Nonetheless, the MOOD loop always converges to an acceptable discrete solution, assuming that the parachute scheme can always produce an acceptable one. In our implementation of MOOD we reconstruct $\mathbb{P}_{M}$ polynomials using a hierarchical polynomial basis. Only one set of neighbor cells (stencil) is used per polynomial degree. As such, the neighborhood to reconstruct a $\mathbb{P}_{3}$ polynomial is wider than the neighborhood for $\mathbb{P}_{1}$ polynomial. No special attention is paid as to carefully choose the stencils, only the standard number of stencil elements is used, see [37].

Moreover, in the DMP $+\mathrm{u} 2$ regularity criteria, contrary to $[29,30]$, we compute the full curvature of the third-order polynomial reconstruction after discarding higher order terms ${ }^{\dagger}$. Let us briefly describe the $\mathrm{DMP}+\mathrm{u} 2$ detection process on a generic variable $A$ and a candidate solution $A_{i}^{*}$ at time $t^{n+1}$ in cell $i$ for a given set of neighbor cells with index $j \in \mathcal{V}_{i}$. The set of vertex neighbors $\mathcal{V}_{i}$ contains all those neighbors of cell $T_{i}$ that have a common vertex with $T_{i}$. First, if $A_{i}^{*}$ fulfills the Discrete Maximum Principle (DMP), that is

$$
\min _{j \in \mathcal{V}_{i}}\left(A_{j}^{n}, A_{i}^{n}\right) \leq A_{i}^{*} \leq \max _{j \in \mathcal{V}_{i}}\left(A_{j}^{n}, A_{i}^{n}\right),
$$

then the cell is valid for this variable. If the DMP is not fulfilled, then one checks the $\mathrm{u} 2$ criterion $[29,30]$, which determines if this new extremum is a smooth extremum, or not. The detection of smooth local extrema has also been discussed in the context of extremum preserving PPM schemes [25] and MPWENO schemes $[4,80]$.

The remaining choices are the detection criteria based on the system of conservation laws to be solved. The purpose of the next section, is to present several criteria depending on the underlying physical processes.

\subsection{MOOD criteria for some particular hyperbolic systems}

This section is devoted to prepare the ADER-MOOD method for three hyperbolic systems that have been chosen to run the validation and verification tests. As already mentioned, for each physical system there are possibly different Physical Admissibility Detection (PAD) criteria as well as Numerical Admissibility Detection (NAD) criteria. Let us consider nonlinear time-dependent hyperbolic systems of conservation laws of the form (2.1).

\footnotetext{
${ }^{\dagger}$ In $[29,30]$ the authors did compute an approximation of the directional second derivatives of the third-order polynomial reconstruction.
} 


\subsubsection{System 1: Hydrodynamics Euler equations (HD)}

The first example are the Euler equations of compressible gas dynamics or hydrodynamics (HD),

$$
\frac{\partial}{\partial t}\left(\begin{array}{c}
\rho \\
\rho \mathbf{v} \\
\rho E
\end{array}\right)+\nabla \cdot\left(\begin{array}{c}
\rho \mathbf{v} \\
\rho \mathbf{v}+p \mathbf{I} \\
\mathbf{v}(\rho E+p)
\end{array}\right)=0
$$

where $\rho$ denotes the mass density, $\mathbf{v}$ the velocity vector, $p$ the fluid pressure, $E$ the total energy density and $\mathbf{I}$ the $d \times d$ identity matrix. With the notation $\mathbf{v v}$ we intend the dyadic product of the velocity vector with itself. To close the system the equation of state (EOS) of a perfect gas is used:

$$
p=(\gamma-1)\left(\rho E-\frac{1}{2} \rho \mathbf{v}^{2}\right)
$$

The pressure is therefore computed as a function of two thermodynamical variables, namely density and the specific internal energy $\varepsilon=E-\frac{1}{2} \mathbf{v}^{2}$ and a gas constant $\gamma$ (ratio of specific heats). Physics demands that density and specific internal energy remain positive so that pressure and sound-speed are un-ambiguously determined. As a consequence the PAD criteria will check that for any discrete (candidate) solution the density and pressure remain strictly positive. For the NAD criteria, following $[18,29,30]$, we choose the DMP + u2 detection criteria but contrarily to the authors who only check the density, we have decided to perform the detection for each conservative variable $\rho, \rho \mathbf{v}, \rho E$. Last, we add the check for impossible discrete values, the famous but annoying NaN (Not-a-Number).

\subsubsection{System 2: Ideal magneto-hydrodynamics (MHD)}

Next, let us consider a more complicated hyperbolic system, namely the equations of ideal magnetohydrodynamics (MHD) in multiple space dimensions. The MHD system introduces an additional difficulty, since the divergence of the magnetic field vector $\mathbf{B}$ must remain zero for all times, i.e.

$$
\nabla \cdot \mathbf{B}=0
$$

For the exact solution of the problem this is always satisfied if the initial data for $\mathbf{B}$ are divergence-free. From the discrete point of view this is not necessarily guaranteed and hence extra care is required in the discretization. In this article we use the hyperbolic version of the generalized Lagrangian multiplier (GLM) divergence cleaning approach proposed in [27]. It consists in adding an auxiliary variable $\psi$ and one linear scalar PDE to the MHD system to transport divergence errors out of the computational domain with the artificial divergence cleaning speed $c_{h}$. The augmented MHD system with hyperbolic GLM divergence cleaning reads

$$
\frac{\partial}{\partial t}\left(\begin{array}{c}
\rho \\
\rho \mathbf{v} \\
\rho E \\
\mathbf{B} \\
\psi
\end{array}\right)+\nabla \cdot\left(\begin{array}{c}
\rho \mathbf{v} \\
\rho \mathbf{v v}+\left(p+\frac{1}{8 \pi} \mathbf{B}^{2}\right) \mathbf{I}-\frac{1}{4 \pi} \mathbf{B B} \\
\mathbf{v}\left(\rho E+p+\frac{1}{8 \pi} \mathbf{B}^{2}\right)-\mathbf{v} \cdot \frac{1}{4 \pi} \mathbf{B B} \\
\mathbf{B v}-\mathbf{v} \mathbf{B}+\psi \mathbf{I} \\
c_{h}^{2} \mathbf{B}
\end{array}\right)
$$

The equation of state (EOS) is now

$$
p=(\gamma-1)\left(\rho E-\frac{1}{2} \rho \mathbf{v}^{2}-\frac{1}{8 \pi} \mathbf{B}^{2}\right)
$$


Several physical constraints for a numerical solution to be admissible are further deduced. First, the density $\rho$ and the pressure $p$ must always remain positive, as for the Euler equations discussed before. Note that the extra magnetic term $-\frac{\mathbf{B}^{2}}{8 \pi}$ in the equation of state leads to a difficult $p>0$ constraint in the case of a highly magnetized flow field. For the numerical detection criteria, as for the Euler system we choose the $\mathrm{DMP}+\mathrm{u} 2$ detection criteria on each conservative variable. Last, we also check for impossible discrete values $(\mathrm{NaN})$.

\subsubsection{System 3: Ideal relativistic magneto-hydrodynamics (RMHD)}

The ideal relativistic MHD (RMHD) equations form a very complicated hyperbolic system, for which the Cauchy-Kovalewski (Lax-Wendroff) procedure as proposed in $[38,40,44,82]$ becomes impossible for orders greater than two. The reason for that is that the primitive variables (and hence the flux tensor $\mathbf{F}$ ) can not be expressed any more in a closed analytical form in terms of the conserved quantities. However, the local space-time Galerkin predictor scheme used in the latest generation of ADER schemes is sufficiently simple and general to be able to deal even with such a complex system as RMHD. The details about this very interesting but very complex hyperbolic system can be found in $[3,47,54,72,96]$. For the multi-dimensional version of the equations, we also have to enforce the divergence-free condition of the magnetic field. As in the non-relativistic MHD case this is done using the hyperbolic divergence-cleaning approach [27]. The vector of conserved variables of the RMHD system reads

$$
\mathbf{Q}=\left(\begin{array}{c}
D \\
\mathbf{q} \\
E \\
\mathbf{B} \\
\psi
\end{array}\right)=\left(\begin{array}{c}
\gamma \rho \\
\gamma w_{t o t} \mathbf{v}-b^{0} \mathbf{b} \\
\gamma^{2} w_{t o t}-b^{0} b^{0}-p_{t o t} \\
\mathbf{B} \\
\psi
\end{array}\right)
$$

and the flux tensor $\mathbf{F}(\mathbf{Q})$ is given by

$$
\mathbf{F}=\left(\begin{array}{c}
\gamma \rho \mathbf{v} \\
\gamma^{2} w_{t o t} \mathbf{v} \mathbf{v}-\mathbf{b} \mathbf{b}+p_{t o t} \mathbf{I} \\
\gamma^{2} w_{t o t} \mathbf{v}-b^{0} \mathbf{b} \\
\mathbf{v B}-\mathbf{B v}+\psi \mathbf{I} \\
c_{h}^{2} \mathbf{B}
\end{array}\right) .
$$

Here, $\mathbf{I}$ is the identity matrix, the enthalpy $w_{t o t}$ and the total pressure $p_{t o t}$ are defined as

$$
w_{t o t}=e+p+|b|^{2}, \quad p_{t o t}=p+\frac{1}{2}|b|^{2},
$$

where the internal energy is given by the following equation of state

$$
e=\rho+\frac{p}{\Gamma-1} \text {. }
$$

The Lorenz factor is

$$
\gamma=\frac{1}{\sqrt{1-\mathbf{v}^{2}}}
$$

and the other quantities appearing in (3.8) are

$$
b^{0}=\gamma(\mathbf{v} \cdot \mathbf{B}), \quad \mathbf{b}=\frac{\mathbf{B}}{\gamma}+\gamma \mathbf{v}(\mathbf{v} \cdot \mathbf{B}), \quad|b|^{2}=\frac{\mathbf{B}^{2}}{\gamma}+(\mathbf{v} \cdot \mathbf{B})^{2} .
$$


We assume a speed of light normalized to unity. The computation of the primitive variables, namely $\mathbf{W}=\{(\rho, \mathbf{v}, p, \mathbf{B}), \psi\}$ from the conserved quantities $\mathbf{Q}$ has to be done numerically, by using an iterative Newton or bisection method, as explained in [33,96].

We can exhibit for this system several physical constraints for a numerical solution to be admissible: First, the density $\rho$ and the pressure $p$ must remain positive. Second, the velocity can never exceed the speed of light (normalized to unity), therefore $|\mathbf{v}|<1$. For the numerical criteria, as for the previous systems we choose the DMP + u2 detection criteria on each conservative variable. Since for RMHD the primitive variables must be computed from the conserved quantities $\mathbf{Q}$ by an iterative method that is not always guaranteed to converge, we also use the failure of convergence of the iterative computation $\mathbf{W}=\mathbf{W}(\mathbf{Q})$ as a numerical detection criterion that enforces a local reduction of the polynomial degree of the reconstruction. Last, we also check for impossible discrete values (NaN).

\subsection{Summary of detection criteria}

In Table 1 we summarize the detection criteria enforced by the physics underlying the three systems of equations.

\begin{tabular}{|c|c|c|c|c|c|}
\hline \multicolumn{6}{|c|}{ MOOD detection criteria } \\
\hline Name & Hyperbolic system & $\begin{array}{r}\text { Phys } \\
\text { D }\end{array}$ & $\begin{array}{l}\text { Amissibility } \\
\text { n (PAD) }\end{array}$ & $\begin{array}{r}\text { Numeric } \\
\text { Dete } \\
\text { for all con }\end{array}$ & $\begin{array}{l}\text { missibility } \\
\text { (NAD) } \\
\text { ve variables }\end{array}$ \\
\hline HD & hydrodynamics & $\rho>0$ & $p>0$ & $\mathrm{DMP}+\mathrm{u} 2$ & $\mathrm{NaN}$ \\
\hline MHD & magneto-hydrodynamics & $\rho>0$ & $p>0$ & $\mathrm{DMP}+\mathrm{u} 2$ & $\mathrm{NaN}$ \\
\hline RMHD & $\begin{array}{c}\text { relativistic } \\
\text { magneto-hydrodynamics }\end{array}$ & $\rho>0$ & $\begin{array}{l}p>0 \\
<1\end{array}$ & $\begin{array}{r}\mathrm{DMP}+\mathrm{u} 2 \\
\mathrm{~W}=\end{array}$ & $\begin{array}{l}\mathrm{NaN} \\
\text { failure }\end{array}$ \\
\hline
\end{tabular}

Table 1: Summary of the detection criteria for the MOOD method for each hyperbolic system of equations which are considered in this work. DMP: Discrete Maximum Principle check, u2: curvature regularity check [29,30], NaN: Not-a-Number check.

\section{Numerical results}

The goals of this section on numerical test cases can be summarized as follows.

1. First we want to show numerically that the coupling of MOOD and ADER techniques provides an accurate and robust numerical method in 2D and 3D for unstructured meshes made of triangles and tetrahedra when smooth and non-smooth problems are simulated;

2. Second, we show that this method is efficient in terms of CPU time, accuracy and memory consumption without any code optimization or subtle tricks. The comparison is made between ADER-MOOD $\mathbb{P}_{3} \longrightarrow \mathbb{P}_{1}^{\text {lim }} \longrightarrow \mathbb{P}_{0}$ and ADER-WENO $\mathbb{P}_{0} \mathbb{P}_{3}$. These performances provide a subjective, but nonetheless meaningful measure: both methods are implemented in the same environment, overheads due to input/output, initialization, MPI parallelization, and other implementation details are shared and can therefore be negligible in the overall count.

3. Third we want to show that MOOD paradigm can adapt to different and more complex systems of conservation laws, namely hydrodynamics (HD), classical Newtonian MHD (MHD) and relativistic MHD (RMHD). As such, one expects to show that genuinely complex physics can 
be handled by the MOOD paradigm. Moreover, the ADER-MOOD framework being almost non-intrusive, its implementation within an existing three-dimensional parallel simulation code is fairly simple, if not almost immediate.

If not stated otherwise in the following we call ADER-MOOD or simply MOOD the $\mathbb{P}_{M} \longrightarrow \mathbb{P}_{1}^{\text {lim }} \longrightarrow \mathbb{P}_{0}$ scheme described in the previous section using conservative variables. The name ADER-WENO or simply WENO is used to refer to the $\mathbb{P}_{0} \mathbb{P}_{M}$ scheme with polynomial WENO reconstruction in characteristic variables according to [37,38]. To our knowledge, this is the most up-to-date unstructured WENO scheme, together with the mixed-element WENO method described in [85,92], which uses the same underlying philosophy. The tetrahedral meshes used in this section are constructed with an automatic mesh generator specifying the domain boundary and a characteristic length called $h$.

\subsection{Numerical results for the HD system}

In this section on the Euler equations of compressible gas dynamics one first uses the isentropic vortex in motion to verify that optimal orders of convergence are reached. CPU time and memory consumption are also provided. Then the classical 2D double Mach reflection problem is run to assess the efficiency of the methods when strong shock waves are present and refined 2D meshes are used. Moreover, Rusanov and HLLE (Harten-Lax-van Leer $[45,51]$ ) fluxes are tested within ADER-MOOD and ADER-WENO methods to enlight the importance of the Riemann solver. Next, the Sod and Lax shock tube problems are run on 3D tetrahedral meshes to show the behavior of the scheme when simple waves are traveling inside the domain without interacting. Finally, a spherical explosion problem is simulated. The purpose of this last test is to show that the conclusions drawn in 2D do also apply in $3 \mathrm{D}$ with even more strength.

\subsubsection{Isentropic vortex in motion}

The isentropic vortex problem was initially introduced for the two-dimensional space [77] to test the accuracy of numerical methods since the exact solution is smooth and has an analytical expression. Let us consider the computational domain $\Omega=[-5,5] \times[-5,5]$ and an ambient flow characterized by $\rho_{\infty}=1.0, u_{\infty}=1.0, v_{\infty}=1.0, w_{\infty}=0.0, p_{\infty}=1.0$, with a normalized ambient temperature $T_{\infty}^{*}=1.0$ computed with the perfect gas equation of state and $\gamma=1.4$.

A vortex is centered on the $z$ axis line at $\left(x_{\text {vortex }}, y_{\text {vortex }}\right)=(0,0)$ and supplemented to the ambient gas at the initial time $t=0$ with the following conditions $u=u_{\infty}+\delta u, v=v_{\infty}+\delta v, w=w_{\infty}, T^{*}=T_{\infty}^{*}+\delta T^{*}$ where

$$
\delta u=-y^{\prime} \frac{\beta}{2 \pi} \exp \left(\frac{1-r^{2}}{2}\right), \quad \delta v=x^{\prime} \frac{\beta}{2 \pi} \exp \left(\frac{1-r^{2}}{2}\right), \quad \delta T^{*}=-\frac{(\gamma-1) \beta}{8 \gamma \pi^{2}} \exp \left(1-r^{2}\right),
$$

with $r=\sqrt{x^{\prime 2}+y^{\prime 2}}$ and $x^{\prime}=x-x_{\text {vortex }}, y^{\prime}=y-y_{\text {vortex }}$. The vortex strength is given by $\beta=5.0$ and the initial density follows the relation

$$
\rho=\rho_{\infty}\left(\frac{T^{*}}{T_{\infty}^{*}}\right)^{\frac{1}{\gamma-1}}=\left(1-\frac{(\gamma-1) \beta}{8 \gamma \pi^{2}} \exp \left(1-r^{2}\right)\right)^{\frac{1}{\gamma-1}} .
$$

Periodic boundary conditions are prescribed everywhere, so that at the final time $t_{\text {final }}=10$ the vortex is back to its original position. Note that this problem has a smooth solution and thus could be simulated with effective high-order of accuracy. Consequently we compute the discrete $L^{2}$ and $L^{\infty}$ norm errors between the initial and final piecewise constant data for the density. Successively refined grids made 
of triangles are constructed given a boundary edge length $h=L_{\text {char }} / N_{x}$ starting from $N_{x}=24$ (coarse grid) up to $N_{x}=128$ (fine grid). In Table 2 we report these errors and the corresponding rates of convergence. These data show that both ADER-MOOD and ADER-WENO are effectively 4th order accurate methods. Moreover errors are of the same order. To compare the CPU time we use the CPU time of the unlimited $\mathbb{P}_{0} \mathbb{P}_{3}$ scheme as the basis because this scheme is the fastest method of effective 4th order. The CPU times reported in Table 2 proves that on this problem WENO is $30 \%$ more expensive than the unlimited scheme whereas MOOD is only $7 \%$ more expensive.

Moreover a CPU times and memory comparisons between ADER-MOOD and ADER-WENO have been carried out using a single CPU core of an Intel i7-2600 processor with $3.4 \mathrm{GHz}$ of clock speed and $12 \mathrm{~GB}$ of RAM, in order to assess the pure serial performance, without accounting for the MPI overhead. The memory consumption for the $N_{x}=64$ mesh is $233.55 \mathrm{MB}$ for WENO and $76.10 \mathrm{MB}$ for MOOD, hence a gain of more than 3 in favor of MOOD. We have also measured that the simulation with ADER-WENO method lasts for 314s whereas ADER-MOOD lasts for 276.7s (ratio 1.13 infavor of MOOD), see the synthetic Table 7.

\begin{tabular}{|c|c|c|c|c|c|c|c|c|c|c|}
\hline & \multicolumn{5}{|c|}{ ADER-MOOD $\mathbb{P}_{3} \longrightarrow \mathbb{P}_{1}^{\text {lim }} \longrightarrow \mathbb{P}_{0}$} & \multicolumn{5}{|c|}{ ADER-WENO $\mathbb{P}_{0} \mathbb{P}_{3}$} \\
\hline$N_{x}$ & CPU time & $L^{2}$ error & & $L^{\infty}$ erro & & CPU time & $L^{2}$ error & & $L^{\infty}$ error & \\
\hline 24 & 1.31 & $2.65 \mathrm{E}-02$ & - & $3,33 \mathrm{E}-02$ & - & 1.15 & $4.49 \mathrm{E}-02$ & - & $3.24 \mathrm{E}-02$ & - \\
\hline 32 & 1.00 & $1.08 \mathrm{E}-02$ & 3.12 & $1.29 \mathrm{E}-02$ & 3.29 & 1.25 & $1.13 \mathrm{E}-02$ & 4.78 & $1.17 \mathrm{E}-02$ & 3.54 \\
\hline 64 & 1.07 & $7.19 \mathrm{E}-04$ & 3.91 & $6.76 \mathrm{E}-04$ & 4.26 & 1.28 & $7.23 \mathrm{E}-04$ & 3.97 & $6.76 \mathrm{E}-04$ & 4.11 \\
\hline 128 & 1.06 & 4.14E-05 & 4.12 & 4.19E-05 & 4.01 & 1.30 & 4.14E-05 & 4.13 & 4.19E-05 & 4.01 \\
\hline \multicolumn{3}{|c|}{ Expected order } & 4 & & 4 & & & 4 & & 4 \\
\hline
\end{tabular}

Table 2: $L^{2}$ and $L^{\infty}$ errors and convergence rate for the isentropic vortex problem for the MOOD $\mathbb{P}_{3} \longrightarrow \mathbb{P}_{1}^{\text {lim }} \longrightarrow \mathbb{P}_{0}$ and WENO $\mathbb{P}_{0} \mathbb{P}_{3}$ methods. CPU times are normalized according to the unlimited $\mathbb{P}_{0} \mathbb{P}_{3}$ scheme.

\subsubsection{Le Blanc shock tube}

Next we have run the shock tube of Le Blanc $[13,61]$ also called sometimes the shock tube from hell. This test is an extreme version of shock tube on domain $[-5: 5]$ for a perfect gas equation of state $(\gamma=5 / 3)$ with the following initial data

$$
(\rho, u, p)(\mathbf{x}, t=0)=\left\{\begin{array}{cll}
\left(1.0,0.0,2 / 3 \times 10^{-1}\right) & \text { if } & x<-2 \\
\left(10^{-3}, 0.0,2 / 3 \times 10^{-10}\right) & \text { if } & x \geq-2
\end{array}\right.
$$

The final time is set to $t_{\text {final }}=6$ and an exact solution is derived from [87]. We run this test in $2 \mathrm{D}$ on domain $\Omega=[-5: 5] \times[-0.5: 0.5]$ on the same unstructured mesh (characteristics length $1 / 50$ leading to 56894 triangles). Comparison between ADER-WENO $\mathbb{P}_{0} \mathbb{P}_{2}$ and ADER-MOOD $\mathbb{P}_{2} \longrightarrow \mathbb{P}_{1}^{l i m} \longrightarrow \mathbb{P}_{0}$ is made in Fig. 1 where we display the log of the density, the $x$-component of the velocity and the specific internal energy variables as a function of $x$. Several cuts along horizontal lines are shown to observe if the symmetry along the $y$-direction is preserved. We also propose the figure showing the cell polynomial degrees at the final time. The Le Blanc shock tube is known to be a very difficult problem because of the strong discontinuity and the subsequent strong emerging waves. Initially a strong entropy deposition is needed at the discontinuity to ensure the stability of the computation. This generally leads to a relative important error on specific internal energy which is further diffused by the numerical method. The more diffusive the numerical scheme, the faster the spike is reduced with an obvious impact on other variables.

Almost any numerical scheme has also difficulties when mesh convergence is demanded. Note that 
the mesh size used here is too large to expect mesh convergence. This is the reason why the position of the shock wave is not well fitted to the exact solution neither for WENO nor for MOOD even if both are conservative methods [81]. In Fig. 1 we can see the classical misbehavior of both methods on internal energy with a more pronounced spike for MOOD. Note that Lagrangian numerical schemes or high-order Eulerian schemes present some defect on internal energy (spike or wrong wave speed) unless specific treatment is designed, see as instance [71]. In the same figure we can observe for the last time step which cells have been decremented (red cells are updated with degree 2, green cells with degree 1 , blue cells with degree 0 ). Most of cells are updated with the $\mathbb{P}_{2}$ reconstructions apart from three zones corresponding to the foot of the rarefaction (marginally), the contact and more pronounced ahead of the shock wave.

\subsubsection{D double Mach reflection of a strong shock}

Next we have run the 2D double Mach reflection of a strong shock that was proposed in [95]. This test problem involves a Mach 10 shock in a perfect gas with $\gamma=1.4$ which hits a $30^{\circ}$ ramp with the $x$-axis. Using Rankine-Hugoniot conditions we can deduce the initial conditions in front of and after the shock wave

$$
(\rho, u, v, p)(\mathbf{x}, t=0)=\left\{\begin{array}{cll}
(8.0,8.25,0.0,116.5) & \text { if } & x<0.1 \\
(1.4,0.0,0.0,1.0) & \text { if } & x \geq 0.1
\end{array}\right.
$$

Reflecting wall boundary conditions are prescribed along the ramp and the upper boundary. Inflow boundary condition and outflow condition are prescribed on the left side and the right side respectively. The same meshes as in [38] using characteristics lengths $h=1 / 200\left(N_{E}=355656\right.$ cells) and $h=1 / 400$ $\left(N_{E}=1418590\right.$ cells $)$ are used. Classically the results are presented as 31 isolines ranging from 1.5 to 21.5 for the density variable at $t_{\text {final }}=0.2$.

Comparison WENO $\mathbb{P}_{0} \mathbb{P}_{3}$ and MOOD $\mathbb{P}_{3} \longrightarrow \mathbb{P}_{1}^{\text {lim }} \longrightarrow \mathbb{P}_{0}$. In Figure 2, we present such isoline contours for WENO and MOOD methods. Furthermore, a zoom on the area of wave interactions is also provided on right panels of Figure 2 to ease the comparison. The results obtained with MOOD present more features than WENO results which indicate that MOOD is less dissipative. As a comparison the reader is referred to [38], where WENO $\mathbb{P}_{2}$ was run on a refined grid $(h=1 / 400)$. Indeed, a somewhat refined grid is needed for the WENO method to be qualitatively comparable to the MOOD method, see for instance the zooms for the $h=1 / 400$ grid.

Moreover a CPU times comparison has been carried out using a grid with characteristic mesh spacing of $h=1 / 100$ on a single CPU core of an Intel i7-2600 processor with $3.4 \mathrm{GHz}$ of clock speed and $12 \mathrm{~GB}$ of RAM, in order to assess the pure serial performance of both schemes, without accounting for the MPI overhead. We have found that for this test case the ADER-WENO method produces a more dissipative solution and is about 1.63 times slower compared to ADER-MOOD, see Table 7. Moreover the memory consumption is 3.41 times less important for MOOD.

Comparison ADER-WENO $\mathbb{P}_{0} \mathbb{P}_{3}$ and ADER-MOOD $\mathbb{P}_{3} \longrightarrow \mathbb{P}_{1}^{\text {lim }} \longrightarrow \mathbb{P}_{0}$ with HLLE numerical flux. In order to show the relative insensitivity of the method to the numerical flux employed we have run the double Mach problem with the HLLE (Harten-Lax-van Leer [45, 51]) numerical flux. This numerical flux was also used in [34] but the underlying ADER-WENO scheme is slightly different (the meshes are not exactly the same but are comparable and also the Cauchy-Kovalewski procedure is not used anymore in the latest generation ADER schemes, like the ones adopted in this paper). The results in Figure 3 are obtained the same way as those of Figure 2 but by using the HLLE flux 

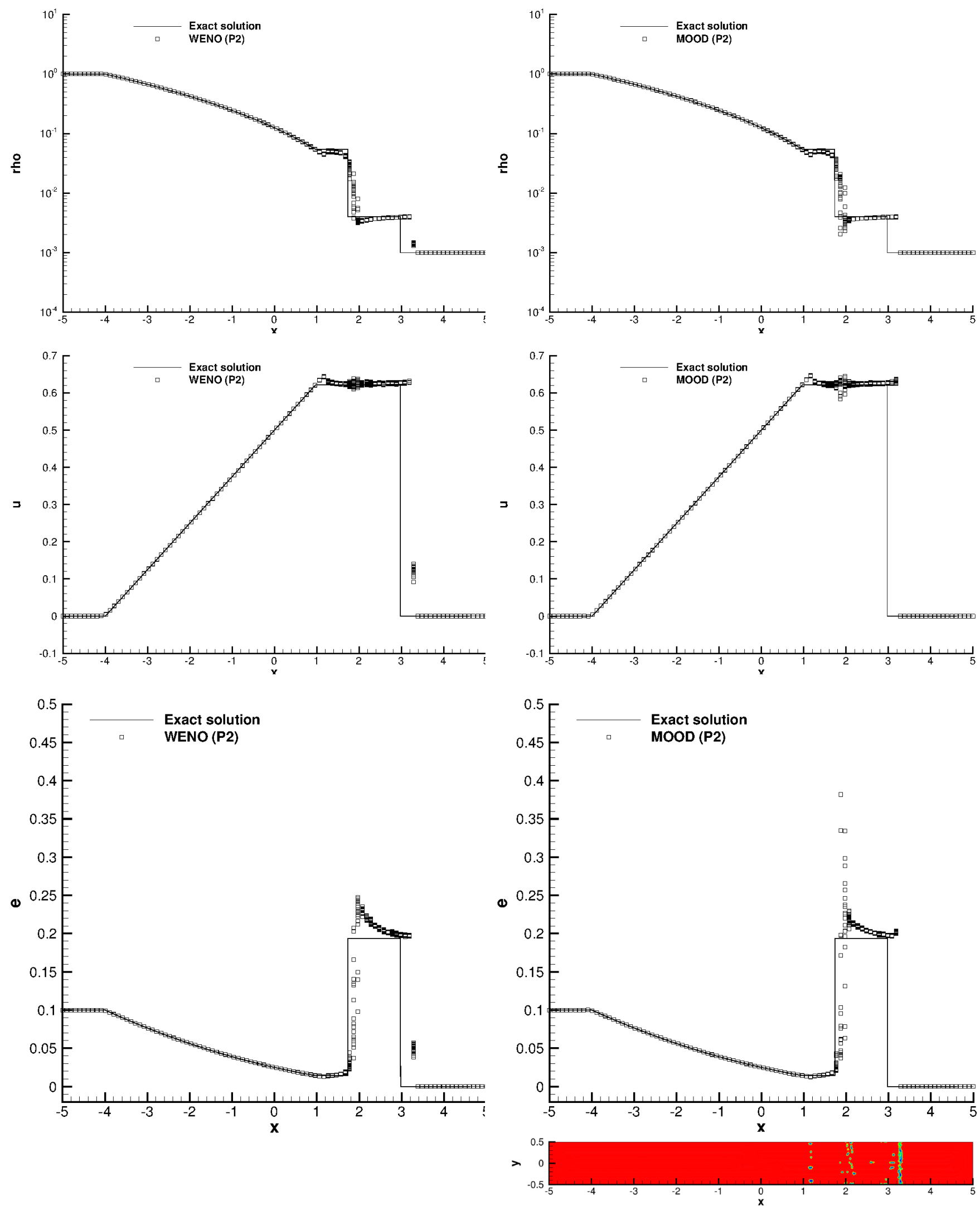

Figure 1: Planar Le Blanc problem at $t_{\text {final }}=6$ on a $2 \mathrm{D}$ unstructured mesh made of 56894 triangles (characteristics length 1/50) - Left row: ADER-WENO $\mathbb{P}_{0} \mathbb{P}_{2}$ results. Right row: ADER-MOOD $\mathbb{P}_{2} \longrightarrow \mathbb{P}_{1}^{\text {lim }} \longrightarrow \mathbb{P}_{0}$ results. $1 \mathrm{D}$ projection along the $O x$ axis of the log of density (top), $x$-component of the velocity (middle) and the specific internal energy (bottom) versus the exact solution. Colored panel: Cell polynomial degrees (red for degree 2, green for 1 and blue for 0 ) 

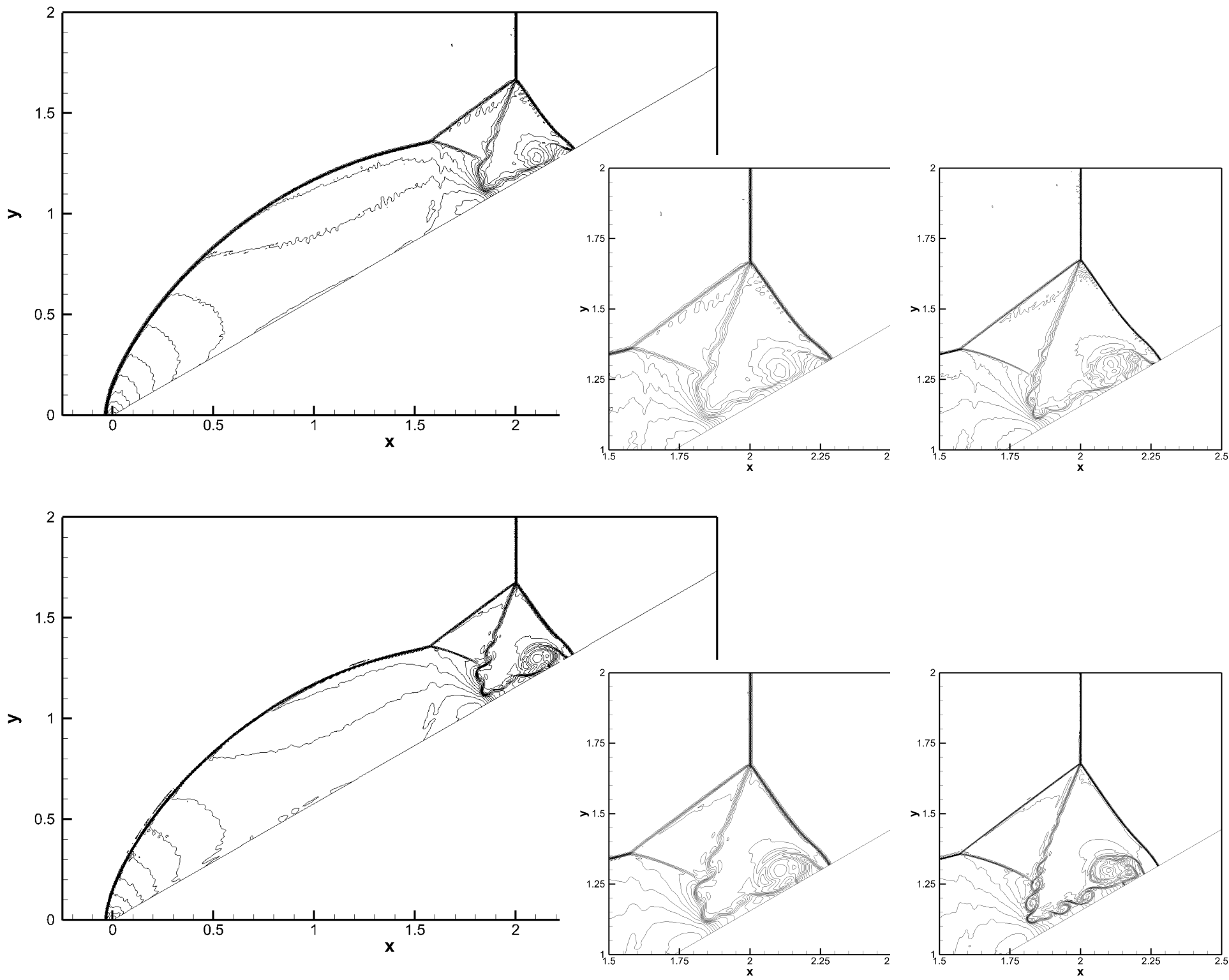

Figure 2: Double Mach problem at $t_{\text {final }}=0.2$ on triangular unstructured meshes - Rusanov numerical flux - Top line: ADER-WENO $\mathbb{P}_{0} \mathbb{P}_{3}$ method. Bottom line: ADER-MOOD $\mathbb{P}_{3} \longrightarrow \mathbb{P}_{1}^{\text {lim }} \longrightarrow \mathbb{P}_{0}$ method - The full domain is shown on the left panel ( $h=1 / 200$ mesh). Zooms for $h=1 / 200$ (middle) and $h=1 / 400$ (right) meshes enhance the results. 


\begin{tabular}{|l||ccc|ccc|c|}
\hline Problem & $\rho_{L}$ & $u_{L}$ & $p_{L}$ & $\rho_{R}$ & $u_{R}$ & $p_{R}$ & $t_{\text {end }}$ \\
\hline \hline Sod & 1.0 & 0.0 & 1.0 & 0.125 & 0.0 & 0.1 & 0.2 \\
\hline Lax & 0.445 & 0.698 & 3.528 & 0.5 & 0.0 & 0.571 & 0.14 \\
\hline
\end{tabular}

Table 3: Initial left and right states for the density $\rho$, velocity $u$ and the pressure $p$ for the planar shock tube problems. The final simulation times $t_{\text {final }}$ are also given.

instead of the Rusanov flux. As such we can observe how WENO and MOOD methods react to the modification of the numerical flux. It seems that HLLE flux is more appropriate for this test case as more structures develop for both methods. Refining the mesh shows the occurrence of more vortices. The conclusions drawn with the Rusanov flux when comparing WENO and MOOD still hold: MOOD method seems to be less dissipative and to produce less oscillatory solutions.

In Figure 4, the cells polynomial degrees map (corresponding to the scheme order minus one) is shown for the last iteration of the MOOD $\mathbb{P}_{3} \longrightarrow \mathbb{P}_{1}^{\text {lim }} \longrightarrow \mathbb{P}_{0}$ method for the two resolutions. As expected the scheme order is maximal almost everywhere except around the principal waves. Some local instabilities which develop behind the waves demand a less accurate scheme to be properly handled, hence the blue/green cells away from principal waves which denote low-order polynomial degrees. Also we observe the presence of the well-known startup error ${ }^{\ddagger}$ manifested by two vertical waves located at $x=0.7$ and $x=1.65$, visible for both methods and all grid resolutions, see Figure 3 . Moreover we remark that around the region where vortices develop (approximatively $x>1.75$ and $y<1.3)$ the MOOD scheme nicely detects that the solution is almost smooth except for few local extrema. As a consequence, this region seems to be updated with the most accurate scheme. This is appropriate should we believe that the solution in this region is smooth enough.

\subsubsection{Planar shock tube problems on 3D unstructured tetrahedral meshes}

Here we run the 1D planar Sod shock tube problem and the classical Lax shock tube problem on a 3D unstructured tetrahedral mesh to assess the ability of the methods to capture one-dimensional simple waves. The initial conditions for density, velocity component $u$ and pressure are listed in Table 3 . The other two velocity components are initialized with $v=w=0$.

The ratio of specific heats is set to $\gamma=1.4$ and for both test problems the discontinuity is initially located at $x=0.0$. An exact solution can be derived for the one-dimensional Riemann problem, see [87] for details. The computational domain $\Omega=[-0.5 ; 0.5] \times[0 ; 0.1] \times[0 ; 0.1]$ is paved with an unstructured mesh made of 190089 tetrahedra constructed with a characteristic mesh size of $h=1 / 100$, leading to an equivalent 100 cell 1D mesh in the principal direction, see Figure 5. Dirichlet boundary condition in $x$ direction are imposed, while in $y$ and $z$ direction we apply periodic boundary conditions. Because the mesh is truly unstructured, 3D effects are to be expected, thus leading to possible loss of symmetry in the transverse directions ( $y$ and $z$ ). In Figures 6 and 7 we present the results for fourth order ADERMOOD and ADER-WENO methods: density, velocity in $x$-direction and pressure are displayed as $1 \mathrm{D}$ cut along the $x$ direction versus the exact solution. Both, ADER-WENO and ADER-MOOD can capture the exact solution rather well. The shock wave is spread across one or two characteristic mesh lengths for both methods, and also the contact discontinuity is resolved in a sharp manner. The star region of the Riemann problem is essentially captured, although some loss of 1D symmetry is observed

\footnotetext{
${ }^{\ddagger}$ The startup error occurs when starting from an isolated traveling shock wave according to the exact Rankine Hugoniot conditions. Since the numerical scheme introduces numerical dissipation in the shock front, spurious waves develop, the so-called startup error. It could be cured by starting with an appropriate discrete shock profile as initial condition.
} 

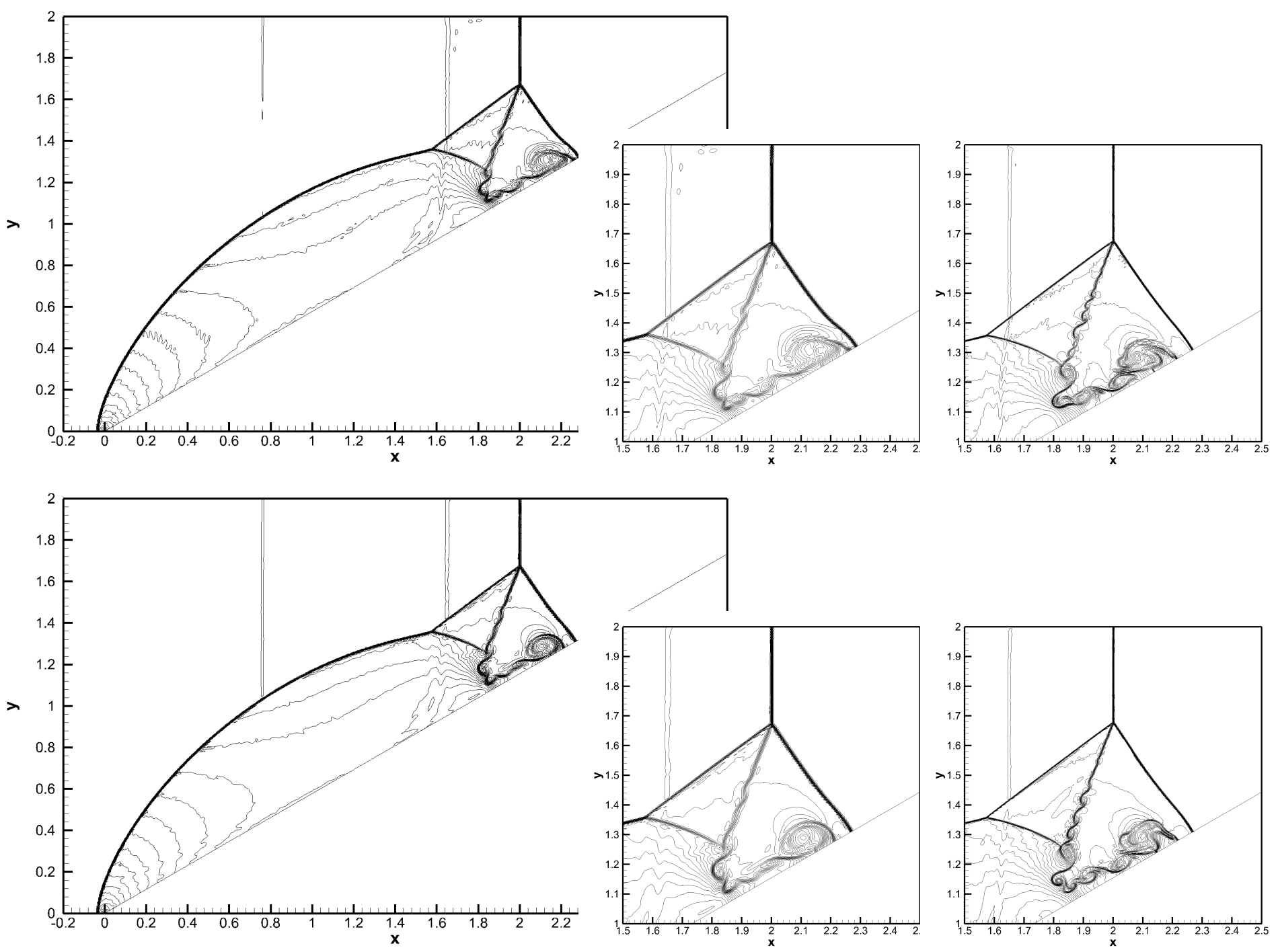

Figure 3: Double Mach problem at $t_{\text {final }}=0.2$ on triangular unstructured meshes - HLLE numerical flux - Top line: ADERWENO $\mathbb{P}_{0} \mathbb{P}_{3}$ method. Bottom line: ADER-MOOD $\mathbb{P}_{3} \longrightarrow \mathbb{P}_{1}^{\text {lim }} \longrightarrow \mathbb{P}_{0}$ method - The full domain is shown on the left panel ( $h=1 / 200$ mesh). Zooms for $h=1 / 200$ (middle) and $h=1 / 400$ (right) meshes enhance the results. 

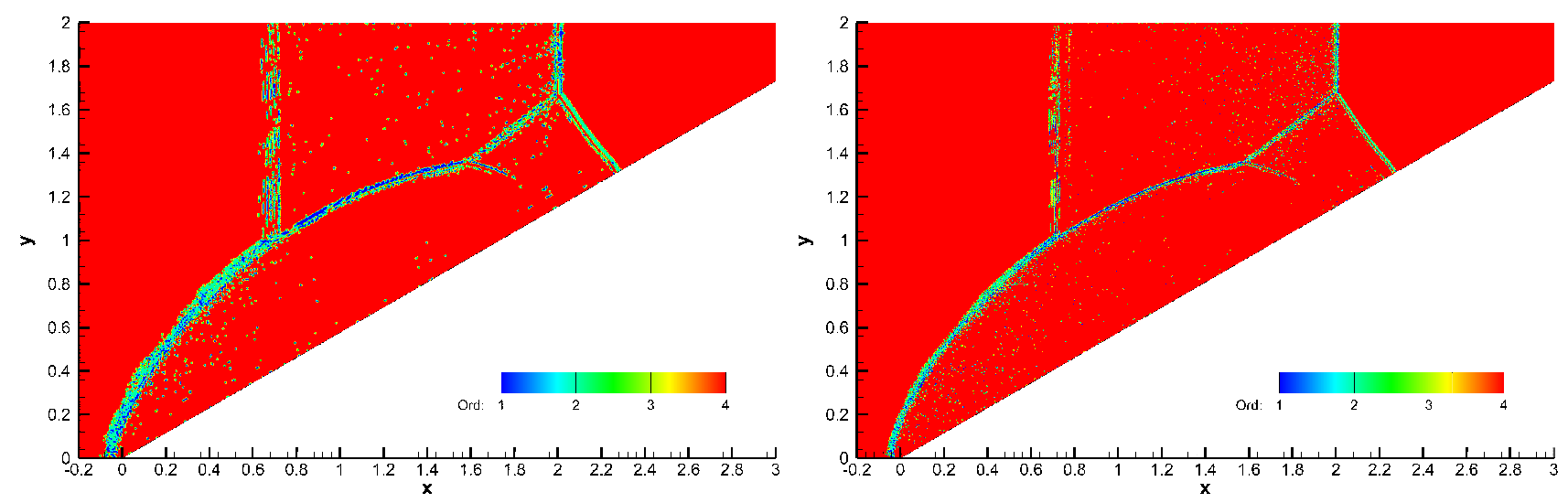

Figure 4: Double Mach problem at $t_{\text {final }}=0.2$ on triangular unstructured meshes - Local order of the scheme (or (CellPD +1$)$ map) ranging from 1 to $4-\mathrm{ADER}-\mathrm{MOOD} \mathbb{P}_{3} \longrightarrow \mathbb{P}_{1}^{\text {lim }} \longrightarrow \mathbb{P}_{0}$ method — Left: $h=1 / 200$ mesh. Right: $1 / 400$ mesh.

\begin{tabular}{|l||ccc|ccc|}
\hline Problem & \multicolumn{3}{|c|}{ ADER-WENO $\mathbb{P}_{0} \mathbb{P}_{3}$} & \multicolumn{4}{|c|}{ ADER-MOOD $\mathbb{P}_{3} \rightarrow \mathbb{P}_{1}^{l i m} \rightarrow \mathbb{P}_{0}$} \\
& $L^{1}$ & $L^{2}$ & $L^{\infty}$ & $L^{1}$ & $L^{2}$ & $L^{\infty}$ \\
\hline \hline Sod & $3.0672 \mathrm{E}-05$ & $9.0131 \mathrm{E}-04$ & 0.1155 & $2.5327 \mathrm{E}-05$ & $7.6584 \mathrm{E}-04$ & 0.1134 \\
\hline Lax & $1.0235 \mathrm{E}-04$ & $4.9910 \mathrm{E}-03$ & 0.7020 & $8.4124 \mathrm{E}-05$ & $4.2497 \mathrm{E}-03$ & 0.7227 \\
\hline
\end{tabular}

Table 4: Errors for the density $\rho$ in three different norms for the fourth order ADER-MOOD and ADER-WENO methods simulating the Sod and Lax problems on a 3D unstructured mesh made of 190089 tetrahedra.

for both methods.

The contact discontinuity seems sharper for MOOD than for WENO. Finally, MOOD presents a slight over- and undershoot at the head and tail of the rarefaction fan. To quantify the errors, we show $L^{1}, L^{2}$ and $L^{\infty}$ error norms for the density for both methods in Table 4. From these results we conclude that ADER-MOOD gives better $L^{1}$ and $L^{2}$ errors, while ADER-WENO provides a better $L^{\infty}$ error for the Lax shock tube problem. For the Sod shock tube, both schemes have essentially the same $L^{\infty}$ error. Considering the absolute value of the errors reached, we can conclude that both high order methods present excellent results on these simple 1D waves.

Finaly, on a single processor, we have measured that MOOD is 1.7 times faster and uses 6 times less memory than WENO, see Table 7 for details.

\subsubsection{D spherical explosion problem}

The last problem is the 3D explosion problem from [87] given by a gas initially at rest in the unit sphere $R=1$. An inside sphere of radius $r_{c}=0.5$ centered at the origin has a density $\rho_{b}=1.0$, a pressure $p_{b}=1.0$ whereas the exterior is initialized by $\rho_{e}=0.125, p_{e}=0.1$ with a perfect gas equation of state with $\gamma=1.4$. The simulation is run up to the final time $t_{\text {final }}=0.2$ on an unstructured mesh made of 3,464 millions of tetrahedra with a characteristic length between $h=0.002$ and $h=0.02$, see Figure 8 to see one hemi-sphere. This problem is run using an MPI parallelized version of the methods on 128 processors. This problem enjoys a spherical symmetry and, as such, a reference solution has been computed using a second-order scheme on the associated 1D partial differential equations with geometric source terms and 10,000 cells, see [87] for details. 


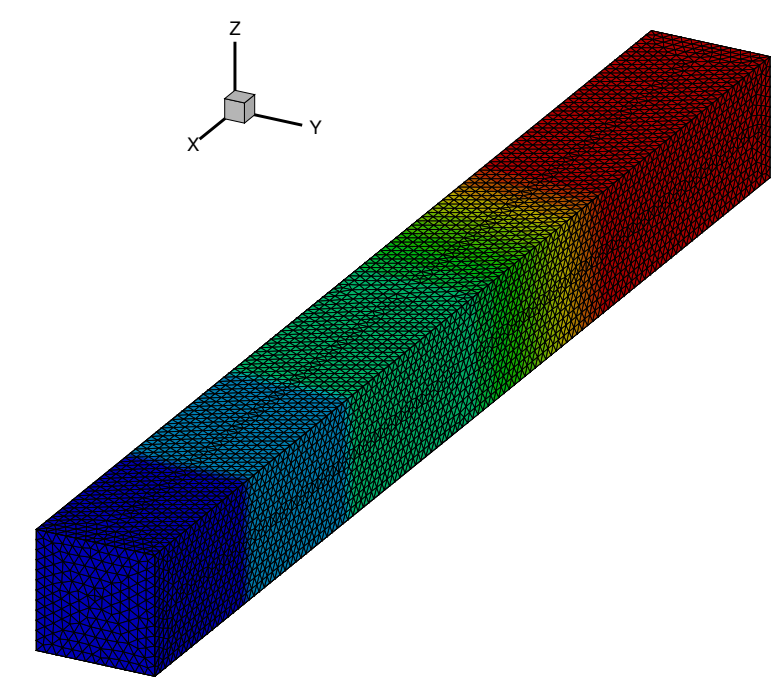

Figure 5: Planar Sod problem at $t_{\text {final }}=0.2$ on a 3D unstructured mesh made of 190089 tetrahedra - MOOD $\mathbb{P}_{3} \longrightarrow \mathbb{P}_{1}^{l i m} \longrightarrow \mathbb{P}_{0}$ results - Mesh and (color) density. Red color corresponds to the unperturbed region to the left, dark blue region to the unperturbed region to the right of the $O x$ axis.

In Figure 9 we present the results for ADER-MOOD (right row) and ADER-WENO (left row) using $\mathbb{P}_{3}$ polynomials versus the reference solution (straight line). The density, velocity ( $u$ component) and pressure are displayed along the positive $x$ axis in the plane $y=z=0$ using 100 equidistant sample points. Both methods are able to compute this spherical solution well, reaching a good level of accuracy. The ADER-MOOD scheme seems to produce a slightly sharper shock wave and contact discontinuity compared to the ADER-WENO method. The rarefaction wave for MOOD, however, presents a small over- and undershoot on the head of the fan, as was already seen on the planar Sod/Lax problems. Also the peak made at the foot of the rarefaction wave (see the velocity, middle panels of Figure 9) seems to be better approximated by MOOD method.

On 512 processors we have measured that MOOD method is 1.28 times faster and uses 3.28 less memory than WENO approach, see Table 7. 

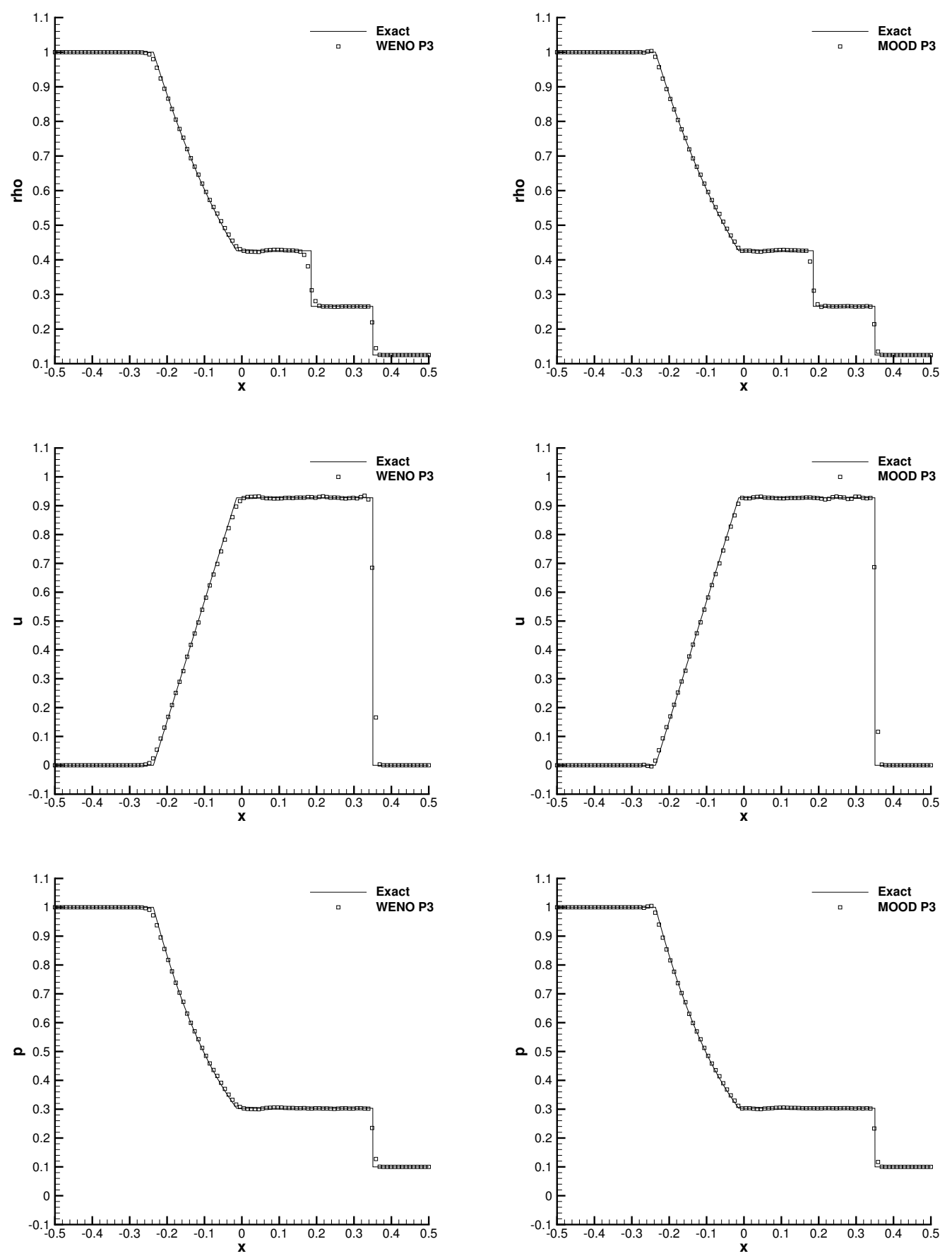

Figure 6: Planar Sod problem at $t_{\text {final }}=0.2$ on a 3D unstructured mesh made of 190089 tetrahedra - Left row: ADER-WENO $\mathbb{P}_{0} \mathbb{P}_{3}$ results. Right row: ADER-MOOD $\mathbb{P}_{3} \longrightarrow \mathbb{P}_{1}^{\text {lim }} \longrightarrow \mathbb{P}_{0}$ results $-1 \mathrm{D}$ projection along the $O x$ axis of the variable (from top to bottom: density, velocity $u$, pressure) versus the exact solution. 

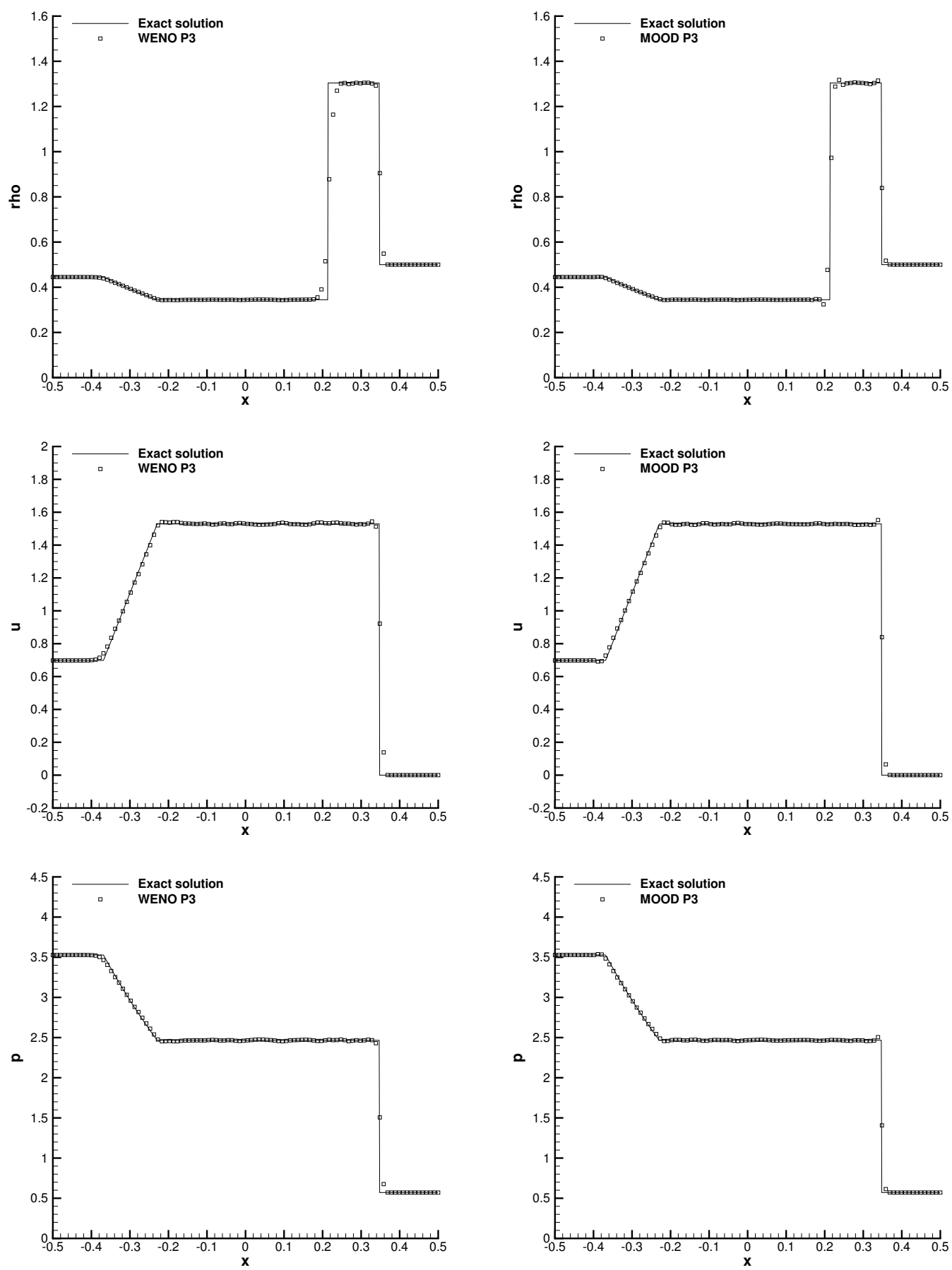

Figure 7: Planar Lax problem at $t_{\text {final }}=0.14$ on a 3D unstructured mesh made of 190089 tetrahedra - Left row: ADER-WENO $\mathbb{P}_{0} \mathbb{P}_{3}$ results. Right row: ADER-MOOD $\mathbb{P}_{3} \longrightarrow \mathbb{P}_{1}^{\text {lim }} \longrightarrow \mathbb{P}_{0}$ results $-1 \mathrm{D}$ projection along the $O x$ axis of the variable (from top to bottom: density, velocity $u$, pressure) versus the exact solution. 


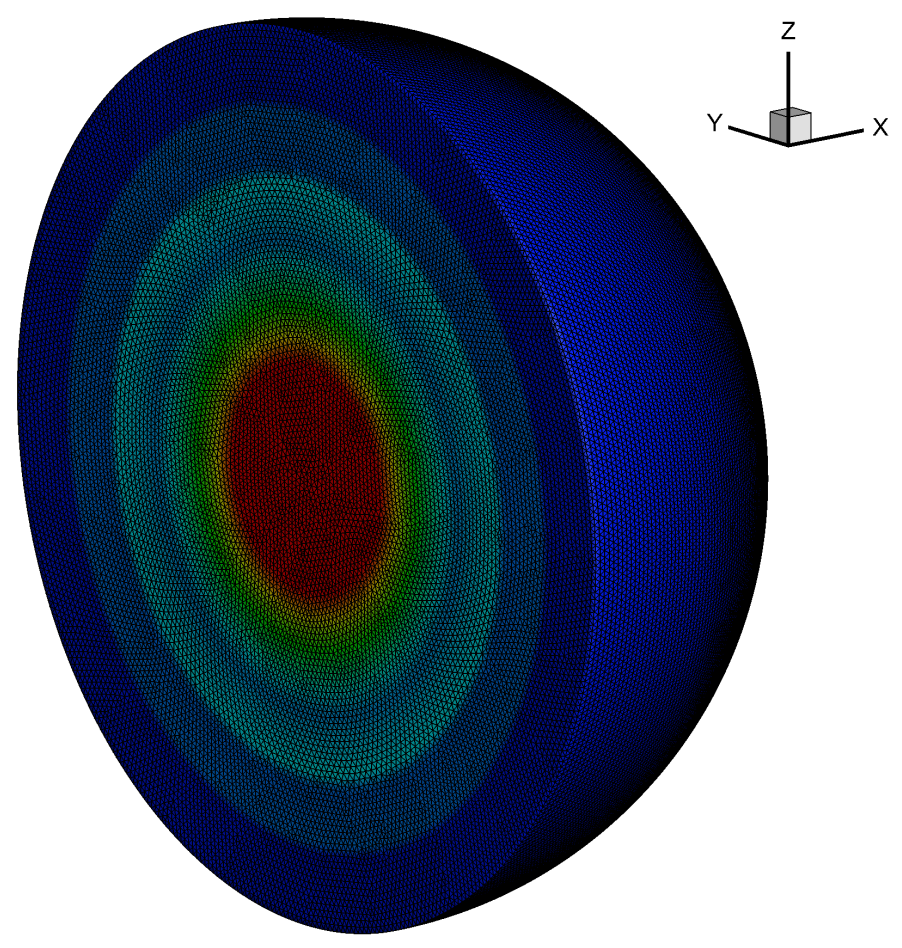

Figure 8: $3 \mathrm{D}$ explosion problem at $t_{\text {final }}=0.2$ on a 3D unstructured mesh made of 3,464 millions of tetrahedra - ADER-MOOD $\mathbb{P}_{3} \longrightarrow \mathbb{P}_{1}^{\text {lim }} \longrightarrow \mathbb{P}_{0}$ results for density (color) on the hemi-sphere. 

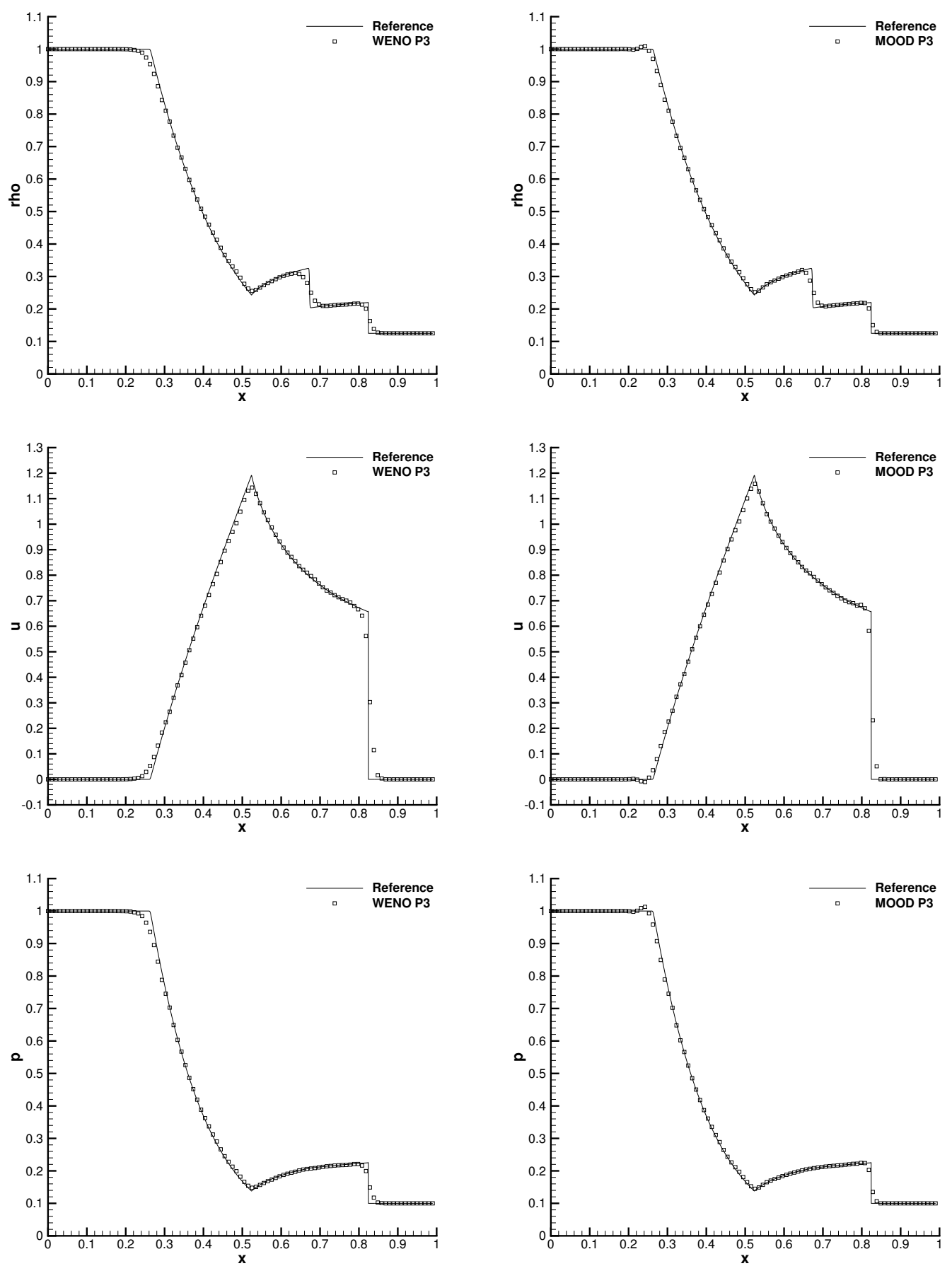

Figure 9: $3 \mathrm{D}$ explosion problem at $t_{\text {final }}=0.2$ on a $3 \mathrm{D}$ unstructured mesh made of 3,464 millions of tetrahedra - Left row: ADER-WENO $\mathbb{P}_{0} \mathbb{P}_{3}$ results. Right row: ADER-MOOD $\mathbb{P}_{3} \longrightarrow \mathbb{P}_{1}^{\text {lim }} \longrightarrow \mathbb{P}_{0}$ results $-1 \mathrm{D}$ projection along the $O x$ axis of the variable (from top to bottom: density, velocity $u$, pressure) versus the reference solution. 


\subsection{Numerical results for the MHD system}

In this section we present the results obtained by the two methods when solving the ideal MHD system in section 3.2.2. The so-called Orszag-Tang Vortex problem [65] and the Rotor problem proposed by Balsara and Spicer in [6] are considered.

\subsubsection{Orszag-Tang vortex system}

The vortex system of Orszag and Tang [65], see also [67] and [26] for more details on the physics of the problem, is now considered. Let us recall the initial data: the computational domain is $\Omega=[0 ; 2 \pi]^{2}$, the same parameters of the computation of Jiang and $\mathrm{Wu}[58]$ are used, however, scaling the magnetic field by $\sqrt{4 \pi}$ due to the different normalization of the governing equations. The initial condition of the problem is given by

$$
\left(\rho, u, v, p, B_{x}, B_{y}\right)=\left(\gamma^{2},-\sin (y), \sin (x), \gamma,-\sqrt{4 \pi} \sin (y), \sqrt{4 \pi} \sin (2 x)\right),
$$

with $w=B_{z}=0$ and $\gamma=\frac{5}{3}$. The final time is $t_{\text {final }}=5.0$. We use a ADER-WENO $\mathbb{P}_{0} \mathbb{P}_{3}$ scheme with reconstruction in conservative variables since the computation of the characteristic variables is too complex and time consuming for this system. Note that MOOD $\mathbb{P}_{3} \longrightarrow \mathbb{P}_{1}^{\text {lim }} \longrightarrow \mathbb{P}_{0}$ still uses reconstruction in conservative variables.

An unstructured triangular mesh with 90126 elements $\left(h=\frac{1}{200}\right)$ is considered. The divergence cleaning speed is set to $c_{h}=2.0$, see [33]. In Figure 10 the results are reported for both methods (WENO on the left column and MOOD on the right) at different output times $t=0.5,2.0,3.0$ and $t_{\text {final }}=5$. Both methods essentially capture the main features and agree with the numerical solutions obtained in [58] and [33], the differences being minor flow details. Comparing the speed and the memory consumption on a single processor we have measured that MOOD is slower than WENO (ratio 0.94 ) but the gain in memory in favor of MOOD is about 2.5, see Table 7 for details.

\subsubsection{MHD Rotor Problem.}

The second test case is the classical MHD rotor problem proposed by Balsara and Spicer in [6]. It consists of a rapidly rotating fluid of high density embedded in a fluid at rest with low density. Both fluids are subject to an initially constant magnetic field. The rotor causes torsional Alfvén waves that are launched into the fluid at rest. As a result the angular momentum of the rotor is diminished. The problem is set up on a circular computational domain $\Omega$ with radius $r=\frac{1}{2}$ The density of the rotor is $\rho=10$ for $0 \leq r \leq 0.1$ and $\rho=1$ for the ambient fluid. The rotor has a constant angular velocity $\omega$ that is determined in such a way to obtain a toroidal velocity of $v=\omega \cdot r=1$ at $r=0.1$. The pressure is $p=1$ in the whole domain and the magnetic field vector is set to $\mathbf{B}=(2.5,0,0)^{T}$ in the whole domain. As proposed by Balsara and Spicer we apply a linear taper to the velocity and density field in the range from $0.1 \leq r \leq 0.13$ such that density and velocity match those of the ambient fluid at rest at a radius of $r=0.13$. The speed for the hyperbolic divergence cleaning is set to $c_{h}=2$ and $\gamma=1.4$ is used. Transmissive boundary conditions are applied at the outer boundaries of the disk. We use a locally refined mesh towards the center of the disk with a total number of 106930 triangles. The characteristic mesh size is $h=0.003$ for $0 \leq r \leq 0.21$ and $h=0.005$ for $0.21<r \leq 0.5$. Results obtained by ADER-WENO and ADER-MOOD are displayed in Figure 11. Density (top line), pressure (middle line) and 'magnetic pressure' $\left(|\mathbf{B}|^{2} /(8 \pi)\right)$ are presented with the same colored scales for each variable to ease the comparison. Both schemes capture the main features of the problem. It seems that MOOD produces smoother profiles than WENO apart from the center of the rotor. We interpret this better behavior by the fact that MOOD is considering only one centered stencil for the polynomial 


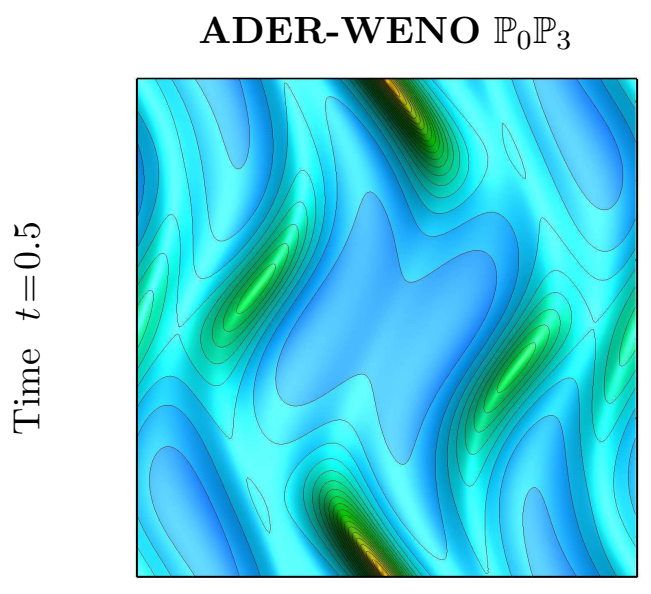

$$
\text { ADER-MOOD } \mathbb{P}_{3} \rightarrow \mathbb{P}_{1}^{l i m} \rightarrow \mathbb{P}_{0}
$$
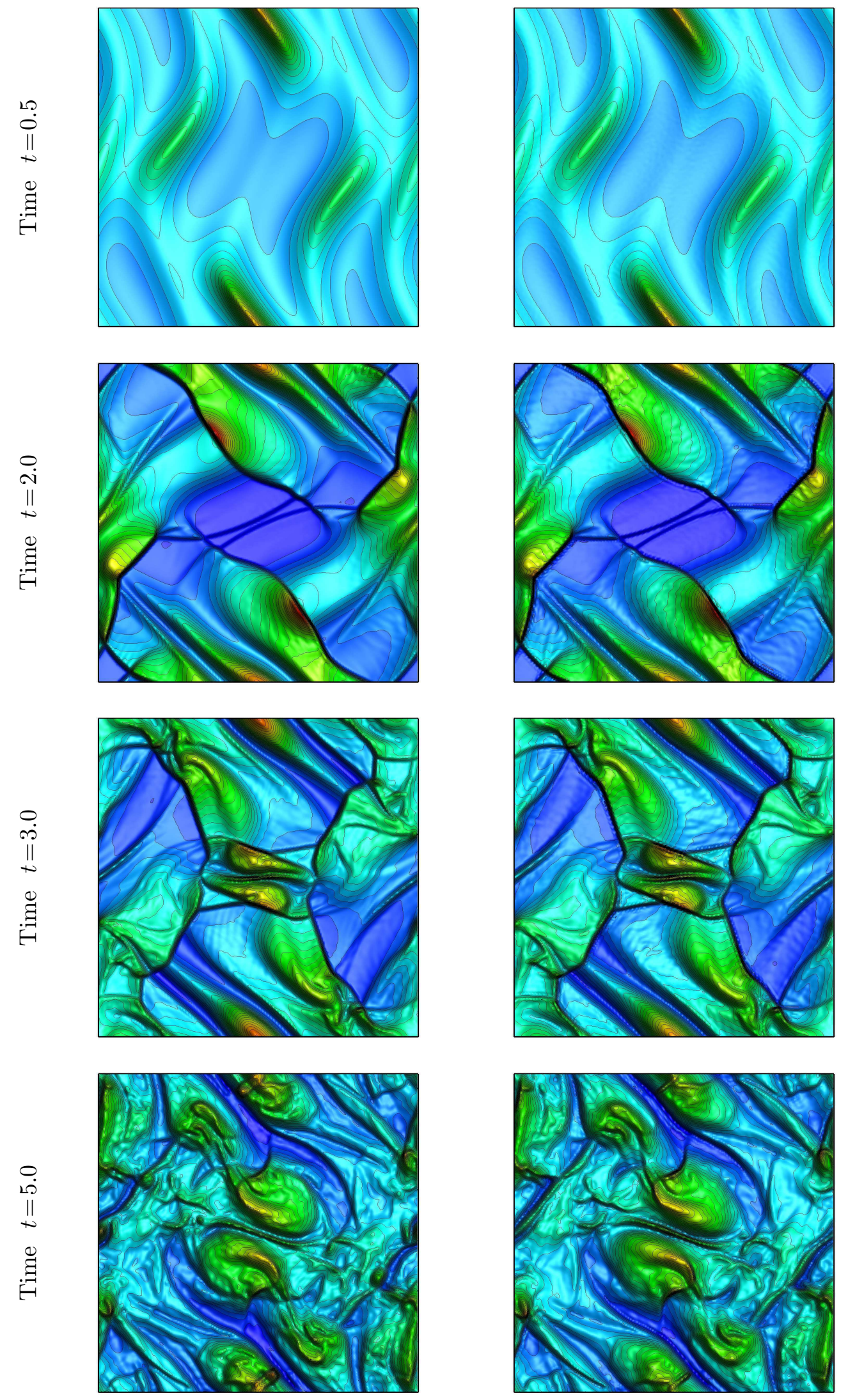

Figure 10: Evolution of the pressure field of the Orszag-Tang problem at times $t=0.5, t=2.0, t=3.0$ and $t=5.0$ (top to bottom) using the ADER-WENO $\mathbb{P}_{0} \mathbb{P}_{3}$ scheme (left column) and ADER-MOOD $\mathbb{P}_{3} \longrightarrow \mathbb{P}_{1}^{\text {lim }} \longrightarrow \mathbb{P}_{0}$ (right column). 
reconstructions for the entire simulation. Contrarily ENO and WENO (in a less pronounced way) consider possibly non-centered stencils which also may change from one time step to the other; this asymmetric treatment has a clear tendency to generate small yet visible numerical instabilities. In the center of the of the rotor, where a plateau seems to be the correct solution the WENO method behaves better because it succeeds in building "almost flat" polynomials. Unfortunately, MOOD does not correct its high-order polynomial if it does not generate a violation of detection criteria. In this almost flat area nothing is invalid after the MOOD cycle, the candidate solution is acceptable as such. For a simulation carried out on a single CPU core for this test MOOD is about 1.14 times faster than WENO and the gain in memory in favor of MOOD is about 2.5, see Table 7 for details. 

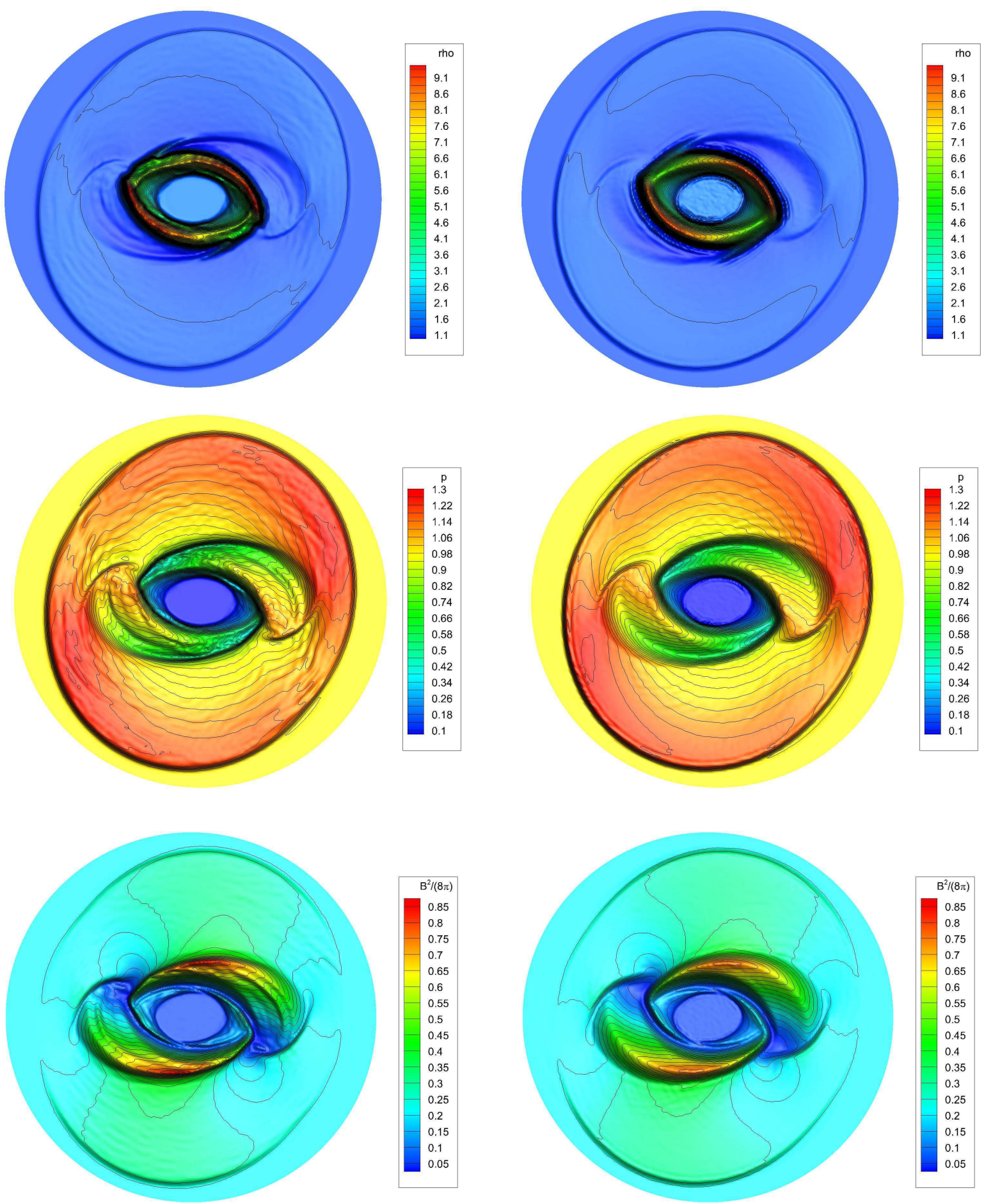

Figure 11: MHD Rotor problem results for the ADER-WENO $\mathbb{P}_{0} \mathbb{P}_{3}$ scheme (left column) and ADER-MOOD $\mathbb{P}_{3} \longrightarrow \mathbb{P}_{1}^{\text {lim }} \longrightarrow \mathbb{P}_{0}$ scheme (right column) - Top line: density. Middle line: pressure. Bottom: magnetic pressure $|\mathbf{B}|^{2} /(8 \pi)$. 


\subsection{Numerical results for the RMHD system}

In this section we present the results obtained by the two methods when solving the resistive relativistic MHD system of equations from section 3.2.3.

\subsubsection{Large Amplitude Alfvén wave.}

The relativistic MHD equations (RMHD) are an extremely challenging and highly nonlinear system of conservation laws. As a direct consequence, it is extremely difficult to devise accurate and robust numerical methods for this system. To verify the accuracy of the proposed high order one-step ADERMOOD finite volume schemes we perform a numerical convergence study of the third, fourth and fifth order version of our schemes on a time-dependent test case originally proposed in the work of Del Zanna et al. in [97]. Subsequently, it has also been used for the validation of other high order schemes in $[33,43,66]$. It consists in a space-time periodic Alfvén wave with large amplitude. The initial condition for the primitive variables is chosen as the exact solution of the problem at time $t=0$. The chosen parameters and the space-time periodic solution are $\rho=p=1, u=B_{x}=\Psi=0, B_{y}=$ $\eta B_{0} \cos \left(k x-v_{A} t\right), B_{z}=\eta B_{0} \sin \left(k x-v_{A} t\right)$ and $v=-v_{A} B_{y} / B_{0}, w=-v_{A} B_{z} / B_{0}$. We use the wavenumber $k=2 \pi$, the 2D computational domain is $\Omega=[0 ; 1] \times[-0.1 ;+0.1]$ with four periodic boundary conditions and $\Gamma=\frac{5}{3}$. With these parameters and $B_{0}=\eta=1$, the speed of the Alfvén wave in positive $x$-direction is $v_{A}=0.433892047069424$, see [97] for details and a closed expression for $v_{A}$. The final computation time is set to $t_{\text {final }}=0.5 \mathrm{In}$ all the computations we use a Courant number of 0.5. Table 5 shows the errors in $L^{2}$ norm, $\epsilon_{L 2}$, the measured convergence orders $\mathcal{O}_{L 2}$ for the flow variable $B_{y}$ and the wallclock time needed for the entire simulation on an AMD Opteron cluster with 64 CPU. The number $1 / h$ denotes the reciprocal characteristic mesh spacing along each coordinate direction and four successively refined meshes are considered.

One can observe that both the ADER-WENO and ADER-MOOD schemes reach their designed order of accuracy, however, the ADER-MOOD method requires sufficiently refined meshes. On coarse grids, the MOOD scheme considers by design that the solution is not resolved enough and as a consequence reduces the polynomial degree of the reconstruction. One also notes that the gains in CPU time are less important for MOOD in this case, since the most expensive part of the scheme is not the reconstruction, but the high order accurate time discretization and the flux calculations. Both require the costly iterative conversion from conservative variables to primitive variables, as outlined above. In addition to that, MOOD requires further extra computations of primitive variables to compute the physical admissibility criteria (PAD), which are based on pressure and density positivity as well as on the norm of the velocity vector, all of which are primitive variables and cannot be easily obtained from the known vector of conserved quantities. The memory consumption for this test for $1 / h=100$ on a single processor is $263.9 \mathrm{MB}$ for WENO and 112.5MB for MOOD; a ratio of 2.3 in favor of MOOD.

\subsubsection{The RMHD blast wave problem.}

This problem is similar to the classical MHD blast wave problem [6] and was also used in the context of resistive RMHD equations in [43]. The initial computational domain is a circle of radius $R_{0}=0.5$ and a mesh with a characteristic mesh size of $h=1 / 400$ and a total number of $N_{E}=282860$ elements is used. The initial condition reads

$$
\mathbf{Q}(\mathbf{x}, 0)=\left\{\begin{array}{lll}
\mathbf{Q}_{i} & \text { if } & r \leq R \\
\mathbf{Q}_{o} & \text { if } & r>R
\end{array}\right.
$$




\begin{tabular}{|c|c|c|c|c|c|c|c|c|c|c|c|}
\hline \multicolumn{4}{|c|}{ ADER-WENO $O 3$} & \multicolumn{4}{|c|}{ ADER-WENO $\mathcal{O} 4$} & \multicolumn{4}{|c|}{ ADER-WENO $\mathcal{O} 5$} \\
\hline $1 / h$ & $\epsilon_{L 2}$ & $\mathcal{O}_{L 2}$ & $t_{\mathrm{CPU}}$ & $1 / h$ & $\epsilon_{L 2}$ & $\mathcal{O}_{L 2}$ & $t_{\mathrm{CPU}}$ & $1 / h$ & $\epsilon_{L 2}$ & $\mathcal{O}_{L 2}$ & $t_{\mathrm{CPU}}$ \\
\hline 50 & $1.066 \mathrm{E}-04$ & - & 11.96 & 25 & 3.644E-05 & - & 5.91 & 25 & $1.165 \mathrm{E}-05$ & - & 10.18 \\
\hline 100 & 1.313E-05 & 3.02 & 86.07 & 50 & $1.576 \mathrm{E}-06$ & 4.53 & 25.90 & 50 & 3.512E-07 & 5.05 & 44.14 \\
\hline 150 & $3.906 \mathrm{E}-06$ & 2.99 & 259.2 & 75 & $2.642 \mathrm{E}-07$ & 4.40 & 55.36 & 75 & 4.625E-08 & 5.00 & 94.66 \\
\hline 200 & $1.650 \mathrm{E}-06$ & 2.99 & 564.9 & 100 & $7.559 \mathrm{E}-08$ & 4.38 & 157.4 & 100 & $1.116 \mathrm{E}-08$ & 4.98 & 273.3 \\
\hline \multicolumn{4}{|c|}{ ADER-MOOD $\mathcal{O} 3$} & \multicolumn{4}{|c|}{ ADER-MOOD $\mathcal{O} 4$} & \multicolumn{4}{|c|}{ ADER-MOOD $O 5$} \\
\hline $1 / h$ & $\epsilon_{L 2}$ & $\mathcal{O}_{L 2}$ & $t_{\mathrm{CPU}}$ & $1 / h$ & $\epsilon_{L 2}$ & $\mathcal{O}_{L 2}$ & $t_{\mathrm{CPU}}$ & $1 / h$ & $\epsilon_{L 2}$ & $\mathcal{O}_{L 2}$ & $t_{\mathrm{CPU}}$ \\
\hline 50 & $8.478 \mathrm{E}-03$ & - & 25.37 & 25 & $1.212 \mathrm{E}-02$ & - & 13.53 & 25 & $1.100 \mathrm{E}-02$ & - & 25.17 \\
\hline 100 & $4.878 \mathrm{E}-03$ & 0.80 & 156.0 & 50 & $1.570 \mathrm{E}-06$ & 12.9 & 25.28 & 50 & 3.510E-07 & 14.9 & 43.07 \\
\hline 150 & 3.907E-06 & 17.6 & 247.4 & 75 & 2.638E-07 & 4.40 & 53.18 & 75 & $4.622 \mathrm{E}-08$ & 5.00 & 91.53 \\
\hline 200 & $1.651 \mathrm{E}-06$ & 2.99 & 533.2 & 100 & 7.516E-08 & 4.38 & 151.3 & 100 & $1.115 \mathrm{E}-08$ & 4.98 & 258.5 \\
\hline
\end{tabular}

Table 5: Numerical convergence study of third, fourth and fifth order unstructured ADER-WENO and ADER-MOOD finite volume schemes for the relativistic MHD equations (RMHD). Errors refer to the variable $B_{y}$.

We use $\gamma=4 / 3$ and the final simulation time is set to $t_{\text {final }}=0.3$. The divergence cleaning speed is $c_{h}=1$. We use the initial condition reported in Table 6 and transmissive boundary conditions are imposed everywhere. We use the third order accurate version of the ADER-WENO finite volume scheme with

\begin{tabular}{|l||ccccccccc|}
\hline & $\rho$ & $u$ & $v$ & $w$ & $p$ & $B_{x}$ & $B_{y}$ & $B_{z}$ & $\Psi$ \\
\hline Inner state $\mathbf{Q}_{i}$ & 1.0 & 0.0 & 0.0 & 0.0 & 1.0 & 0.05 & 0.0 & 0.0 & 0.0 \\
Outer state $\mathbf{Q}_{o}$ & 1.0 & 0.0 & 0.0 & 0.0 & $10^{-3}$ & 0.05 & 0.0 & 0.0 & 0.0 \\
\hline
\end{tabular}

Table 6: Initial condition for the RMHD blast wave problem.

the simple Rusanov-type flux and compare it with a third order ADER-MOOD method on the same grid $(h=1 / 400)$. The numerical results are depicted in Figure 12. The contour colors of the magnetic field component $B_{x}$ are reported (11 color contours are exponentially distributed between 0.03 and 0.3 ) and the numerical results obtained with both schemes agree well with each other.

\subsubsection{RMHD rotor problem}

We propose to solve a relativistic version of the MHD rotor problem of Balsara and Spicer [6]. The test case is exactly the same as proposed in [96]. For this situation the Lorentz factor is very high $(\gamma \approx 10)$ and high order methods may encounter difficulties with pressure positivity. Therefore, a strategy as described in detail in [5] should be applied. For WENO method we must reduce the order of accuracy locally to one in those elements where negative pressures are encountered in the time evolution phase. The rotor has a radius of $R_{i}=0.1$ and is spinning with an angular frequency of $\omega=9.95$, leading to maximal toroidal velocities of $v=0.995$. Recall that the speed of light is normalized to 1 , consequently the maximal toroidal velocity is less than $0.5 \%$ close. The density is $\rho=10$ inside the rotor and $\rho=1$ in the fluid initially at rest. The pressure is $p=1$ and the magnetic field is $\mathbf{B}=(1,0,0)^{T}$ in the entire domain. The speed for the hyperbolic divergence cleaning is set to $c_{h}=1$ and $\Gamma=5 / 3$ is used. Finally transmissive boundary conditions are applied at the outer boundary. The same mesh as for the classical MHD rotor test case is used. We use the $\mathbb{P}_{0} \mathbb{P}_{3}$ ADER-WENO scheme on conservative variables, since the computation of the characteristic variables is too complex and time consuming for this system, and the same ADER-MOOD method as previously. Only the new constraint on the 

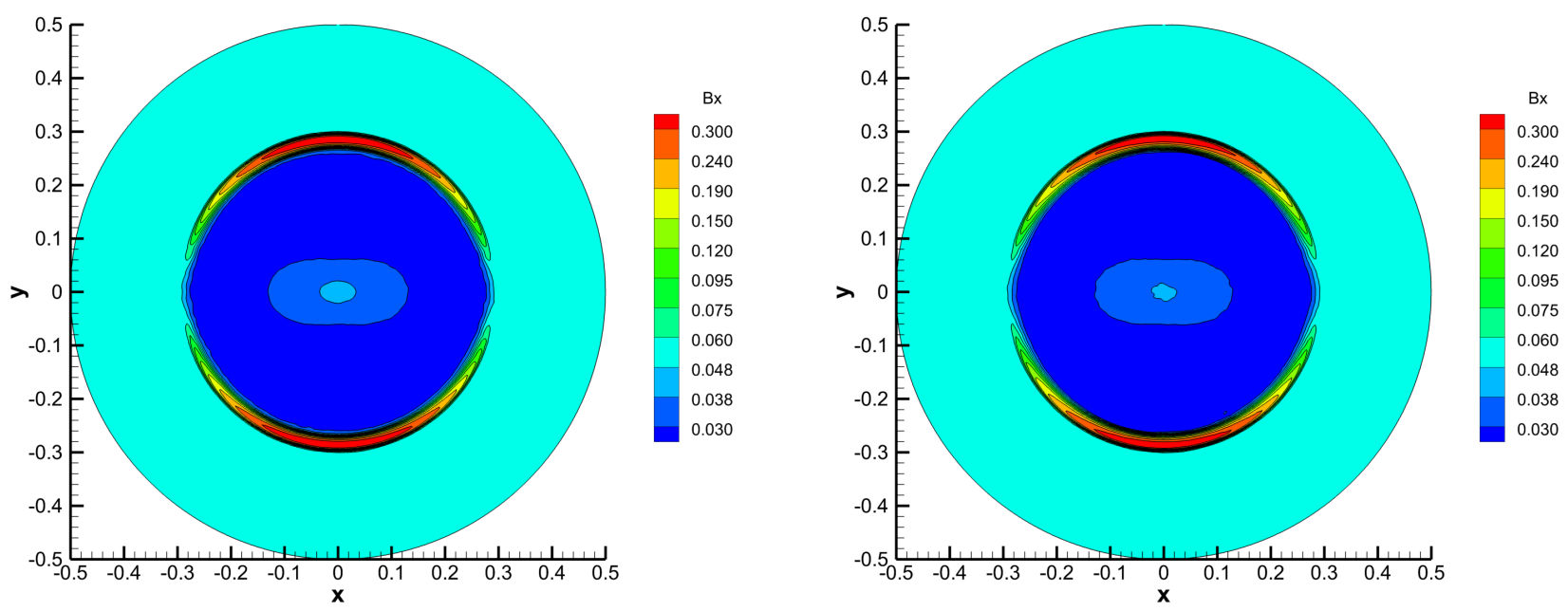

Figure 12: Results for the magnetic field component $B_{x}$ for the RMHD blast wave problem at the final time $t_{\text {final }}=0.3$. 11 color contours are exponentially distributed between 0.03 and 0.3 . Left: Third order ADER-WENO scheme on a grid with $h=1 / 400$. Right: Third order ADER-MOOD scheme on the same grid.

speed of light is added to the set of detection criteria, as well as the failure in the iterative conservative to primitive variable conversion process, see section 3.2.3. The whole computational domain together with the results for density, pressure and magnitude of the magnetic field (in colors and isolines) at final time $t_{\text {final }}=0.4$ are displayed in Figure 13 for ADER-WENO scheme (left column) and ADERMOOD (right column). We observe that the Alfvén waves ejected by the rotor into the medium at rest are well reproduced by both methods. As reported in [96] we also observe a strong roll-up of the shear waves. Qualitatively MOOD seems to be less sensitive to parasitical instabilities than WENO for this very difficult problem. Note that the polynomial degree decrementing implemented for WENO method when negative pressures are encountered is mandatory for the code not to crash. Obviously the MOOD method also needs such constraint, however, this is one of the physical admissible detection (PAD) criteria.

For a simulation carried out on a single CPU core for this test MOOD runs at about the same speed than WENO (ratio 0.96) and the gain in memory in favor of MOOD is about 2.5, see Table 7 for details.

This test somewhat justifies why a posteriori treatment of bad cells is an interesting approach. Indeed this shows that the most up-to-date high-order ADER-WENO method is not a priori positivity preserving for the pressure when the underlying system becomes complex and the test case is demanding. Nonetheless a simple a posteriori correction like the proposed 'trick' cures this flaw. Unfortunately, as it stands, there is no place for such a useful a posteriori trick within the classical WENO philosophy. For more recent developments on a very elegant formulation of positivity preserving WENO schemes, the reader is referred to a recent series of papers $[7,56,98]$ and references therein. However, it is not yet clear how to assure positivity with this new high order WENO approach for the very complex RMHD system. Contrarily, the high order MOOD method is positivity preserving by construction, under the assumption that the parachute scheme is positivity-preserving. 

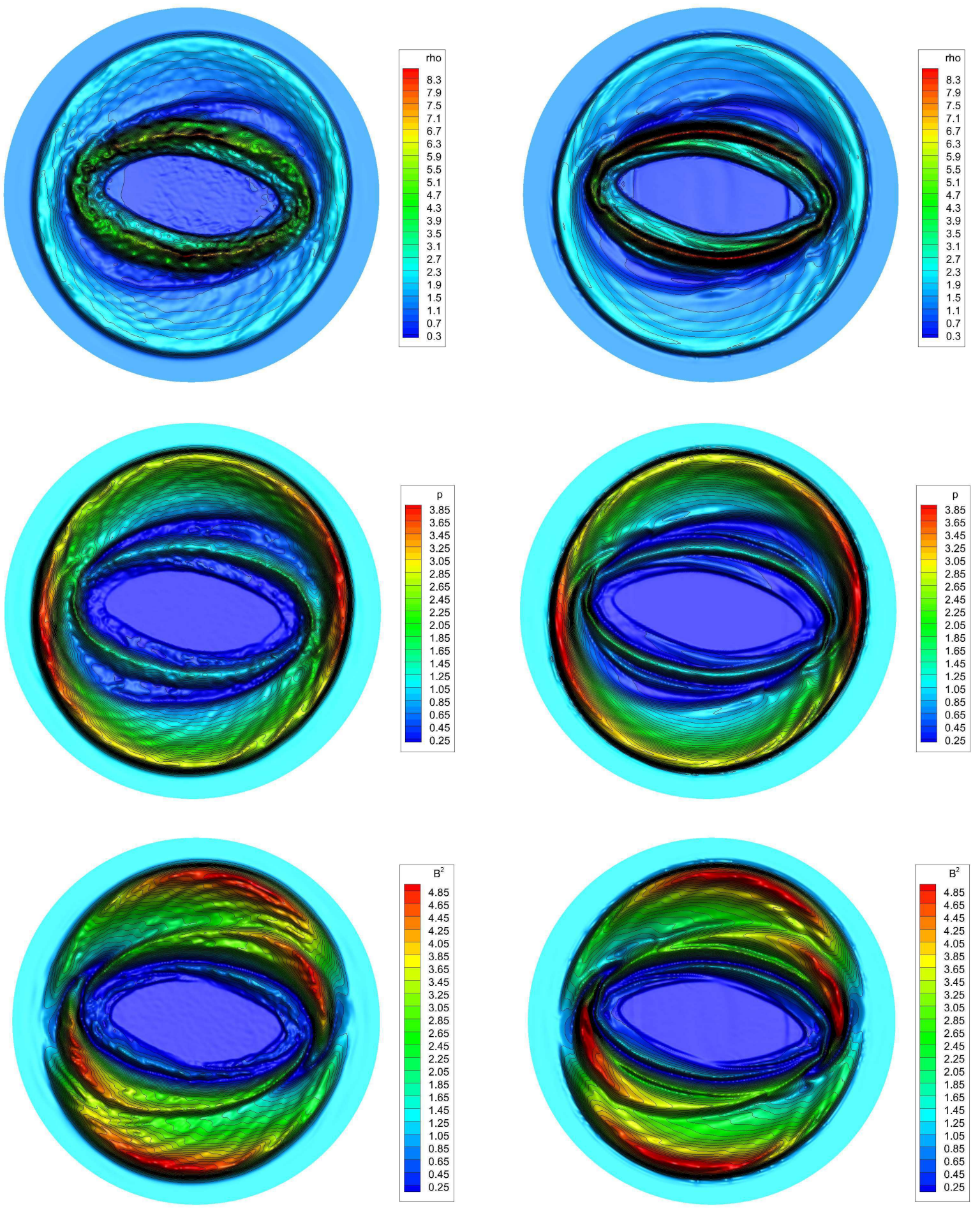

Figure 13: Relativistic MHD Rotor problem results for the ADER-WENO $\mathbb{P}_{0} \mathbb{P}_{3}$ scheme (left column) and ADER-MOOD $\mathbb{P}_{3} \longrightarrow \mathbb{P}_{1}^{\text {lim }} \longrightarrow \mathbb{P}_{0}$ scheme (right column) at $t_{\text {final }}=0.4-$ Top line: density. Middle line: pressure. Bottom: magnetic field $|\mathbf{B}|^{2}$. 


\subsection{CPU time, memory and algorithm}

For the previous test cases and comparisons we have observed that the high order MOOD method often seems to be more accurate, or is at least as accurate as the classical WENO method in most cases. Now it remains to compare the cost of both methods in terms of CPU time and memory consumption. These two measures are equally important: 'CPU time' refers to how much time one should wait for the results, 'memory consumption' refers to how fine can be the mesh resolution because on parallel machines and for scalable implementation the last bottleneck is the memory charge.

In theory we can estimate the possible gain in the reconstruction procedure when comparing WENO and MOOD methods. By 'reconstruction procedure' we mean the time needed to build and store the matrix depending on the chosen stencil, and, the matrix dimensions depend on the polynomial degree. To reconstruct such a polynomial the machine loads the matrix from the memory then inverts it and stores the obtained polynomial coefficients. The matrix storage (memory) and inversion (CPU time) are the two places where MOOD and WENO differ. In 3D WENO $\mathbb{P}_{3}$ demands at least 9 different reconstruction stencils whereas MOOD method reconstructs one $\mathbb{P}_{3}$ polynomial and one limited $\mathbb{P}_{1}$ polynomial. The limited $\mathbb{P}_{1}$ polynomial for MOOD costs less than one WENO $\mathbb{P}_{3}$ reconstruction. As a consequence, for the reconstruction step only, we can expect MOOD to be 8 times more efficient in time and memory than WENO. In 2D this drops to 6 times. However the MOOD loop has an extra-cost which is rather difficult to estimate. Experiments have shown that $10 \%-15 \%$ of invalid cells per time step are usually observed. (Recall that for the isentropic vortex MOOD was $6 \%$ more expensive than the unlimited scheme, WENO was $30 \%$ more expensive.)

For a dedicated code to simulate 3D Euler equations without source terms the cost of the reconstruction overtops all other costs for the WENO method. As a consequence, in such a situation, MOOD is expected to be less expensive than WENO with a ratio no better than 8 times. Around $6-7$ times is presumably a good estimate taking into account the extra-cost of the MOOD loop.

This is different for a non-dedicated code, as instance the code used in this article. Indeed the original code has been designed to solve very general systems of PDEs of the form

$$
\mathbf{Q}_{t}+\nabla \cdot \mathbf{F}(\mathbf{Q}, \nabla \mathbf{Q})+\mathbf{B}(\mathbf{Q}) \cdot \nabla \mathbf{Q}=\mathbf{S}(\mathbf{Q}),
$$

with viscous terms, source terms and non-conservative products all included, some of which demand volume integrals, etc. The relative cost of the polynomial reconstruction procedure drops significantly since the computation of all other complex terms is usually more demanding [34]. Also in such a code the ADER technique demands to keep in memory not only the space-time polynomials of state variables but also the space-time flux tensor, and the algebraic source terms. Therefore the relative cost in memory of the polynomial reconstruction procedure is lower for such a general code. Even if the WENO polynomial reconstruction is still expensive its relative cost to the whole scheme drops to $60 \%$. The $40 \%$ remaining is the incompressible cost of the code that MOOD can not further reduce. Therefore the ratio 8 between MOOD and WENO estimated previously will apply only to $60 \%$ of the overall cost. Hence for $100=60+40$ unit of times for WENO method, MOOD should outperforms with a cost of $60 / 8+40=47.25 \simeq 50$ units of times, that is to say an effective ratio of 2 .

As already mentioned one of our goal in this paper was to show that without any fine tunings and with ultra light developments a MOOD paradigm instantaneously improves the performance of an already existing code solving hyperbolic system of equations. More advanced development into the code would certainly improve the memory consumption and CPU time in favor of MOOD. As such the observed gains are presumably below the maximal expected gains. 


\begin{tabular}{|c|c||ccc|ccc|}
\hline PDEs & Test case & \multicolumn{3}{c||}{ CPU time $[\mathbf{s}]$} & \multicolumn{3}{c|}{ Memory [GB] } \\
& & WENO & MOOD & Ratio & WENO & MOOD & Ratio \\
\hline \hline \multirow{4}{*}{ HD } & 2D Isentropic vortex (1 CPU core) & 313.8 & 276.7 & 1.13 & 233.55 & 76.10 & 3.07 \\
& 2D Double Mach reflection (1 CPU core) & 5425 & 3327 & 1.63 & 2.14 & 0.628 & 3.41 \\
& 3D planar Sod shock tube (1 CPU core) & 46606 & 27283 & 1.70 & 13.44 & 2.464 & 5.98 \\
& 3D Explosion (512 CPU cores, MPI) & 1791.6 & 1399.0 & 1.28 & 460.5 & 140.6 & 3.28 \\
\hline \multirow{2}{*}{ MHD } & 2D Orszag-Tang vortex (1 CPU core) & 15602 & 16599 & 0.94 & 2.536 & 1.002 & 2.53 \\
& 2D MHD rotor (1 CPU core) & 3315 & 2902 & 1.14 & 1.991 & 0.793 & 2.51 \\
\hline RMHD & 2D RMHD rotor (1 CPU core) & 17434 & 18098 & 0.96 & 1.993 & 0.794 & 2.51 \\
\hline
\end{tabular}

Table 7: Wallclock times and memory consumption for WENO and MOOD methods. Also the ratio of computer resource consumption of WENO with respect to MOOD is given.

In Table 7 we present the CPU times and memory consumptions for several test cases provided in this paper and for both methods. (Some of these results have already been previously given and commented.) Some tests have been run on a single processor, some on 64,128 or 512, consequently the number of seconds for the CPU time is not truly informative, only the ratio between the WENO and MOOD performance matters. Here we mainly focus on single CPU core results to avoid the overhead brought by the parallelization.

Finally in Figure 14 we present a sketch of the MOOD loop embracing an existing hyperbolic solver. The solver provides a candidate solution at $t^{n+1}$ which is tested according to the Physical Admissible Detection and Numerical Admissible Detection criteria (blue boxes). If all cells pass the test then the solution as a whole is acceptable and the time step is done. In the case some problematic cells do not pass the tests, they are listed into a set $\mathbf{P}$ and a set $\mathbf{P}^{\prime}$ where their neighbors are also gathered. $\mathbf{P}^{\prime}$ corresponds to the set of cells which need to be recomputed. Next the polynomial degree of any cell in $\mathbf{P}$ is decremented. Accordingly the face polynomial degrees are modified, they correspond to the actual degree of the polynomials used to evaluate the fluxes at cell interfaces, see [29] for the details. This set of few cells is further sent to the solver for recomputation which provides a new candidate solution to be tested. 


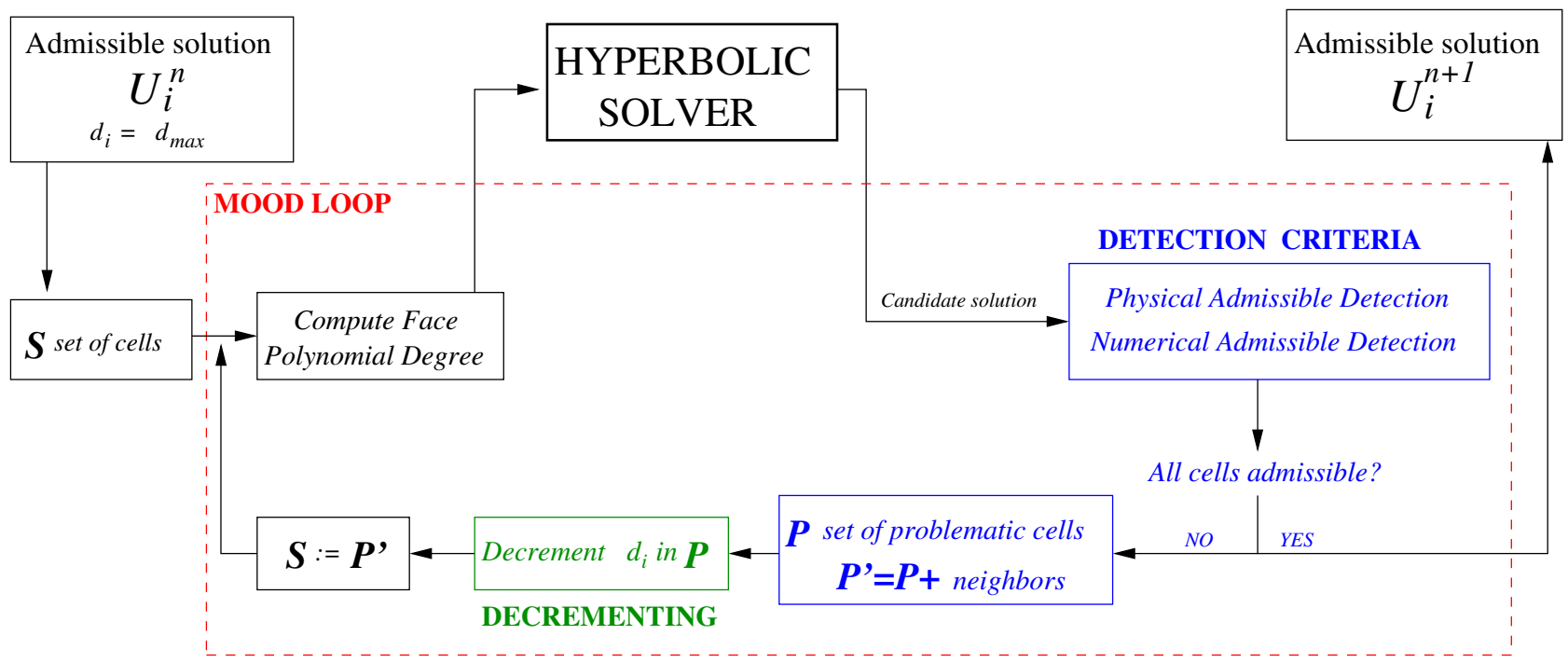

Figure 14: Sketch of the MOOD LOOP embracing an existing hyperbolic solver. The blue boxes correspond to the detection process where a candidate solution in a given cell is declared acceptable or marked as problematic in the set $\mathbf{P}$. The green box performs the decrementing of cell polynomial degree $d_{i}$ for problematic cells which are to be recomputed along with their neighbors. Once every cells are acceptable (or if their polynomial degree has dropped to 0 ) the candidate solution is considered to be acceptable and the timestep is terminated. 


\section{Conclusion and perspectives}

In this work we have presented the coupling of the MOOD concept with a high order one-step ADER discretization for hyperbolic systems of equations on unstructured meshes in two and three space dimensions. The previous a priori WENO type of limitation within the ADER scheme has been replaced by the a posteriori polynomial degree decrementing developed in the MOOD concept. Reciprocally, the ADER technique has replaced the RK3 time discretization in the original MOOD method. As such, we have built a high order one-step space and time discretization method. Let us recall the developer's choices which must be done when a MOOD loop is to be implemented within an existing simulation code:

- Which cascade of schemes is to be used? Here we have chosen the cascade $\mathbb{P}_{M} \longrightarrow \mathbb{P}_{1}^{\text {lim }} \longrightarrow \mathbb{P}_{0}$ with $M=3,4$, or 5 .

- Which Physical Admissibility Detection (PAD) and Numerical Admissibility Detection (NAD) criteria are to be used? The system of partial differential equations usually dictates the PAD whereas the NAD must be designed. Here we have used the so-called DMP $+\mathrm{u} 2$ detection criteria on all conservative variables.

Note that these choices could be different but usually the user/developer has a clear understanding of the system to solve and the properties of the solution to be maintained. This coupling of netween ADER and MOOD was indeed the first purpose of this work.

The second goal was to numerically validate this new approach. The ADER-MOOD scheme has been first implemented and tested on the Euler equations of compressible gas dynamics. Numerical evidences have been provided to assess the effective high-order of accuracy in space and time on smooth solutions but also the accuracy by comparing our results with classical ADER-WENO results. Accuracy, memory and CPU time consumption have also been compared between the two approaches. For the test cases we carried out, the ADER-MOOD scheme requires at least 3 times less memory storage and runs $60 \%$ faster when sequential and 30\% when parallelized with MPI than classical ADER-WENO schemes. Only very little efforts were necessary to implement the MOOD loop into the existing $3 \mathrm{D} \mathbb{P}_{N} \mathbb{P}_{M}$ MPI parallel simulation code. In other words, we have not tried to optimize in any possible way the MOOD procedure to fit neither the existing data-structure nor the parallelization choices. Because this original $\mathbb{P}_{N} \mathbb{P}_{M}$ code is not designed to welcome a MOOD loop the improvement measured in this paper is at minimal level that one could expect - this was also the fourth goal of this paper, to show that MOOD is non-invasive to an existing code.

The fifth goal was to show that the MOOD concept adapt instantaneously to other systems of conservation laws: in this paper we extended it to MHD and relativistic MHD. The underlying physics implies new detection criteria to supplement the already existing listed in the Physical Admissibility Detection set.Numerical validation on known problems were also provided along with a detailed comparison with ADER-WENO results. For the same mesh resolution, computational times are comparable for ADER-MOOD and ADER-WENO but ADER-MOOD produces less oscillatory results for most of the test cases and demands about 2.5 time less memory.

In the near future we plan to investigate how ADER-MOOD behaves when stiff source terms and non-conservative products are present within the system, e.g. for the multi-layer shallow water equations or the Baer-Nunziato model of compressible multi-phase flows. We recall that the simulation code has been designed to solve such generic systems as given by equation (4.6). As a consequence, 
we plan to investigate more thoroughly how the MOOD concept will adapt to more physics based systems, should they be conservative or not.

\section{Acknowledgments}

M.D. has been financed by the European Research Council (ERC) under the European Union's Seventh Framework Programme (FP7/2007-2013) with the research project STiMulUs, ERC Grant agreement no. 278267. R.L. has been partially funded by the ANR under the JCJC project "ALE INC(ubator) 3D". This work has been authorized for publication under the reference LA-UR-13-28795. The authors would like to acknowledge PRACE for awarding access to the SuperMUC supercomputer based in Munich, Germany at the Leibniz Rechenzentrum (LRZ).

\section{References}

[1] R. Abgrall. On essentially non-oscillatory schemes on unstructured meshes: analysis and implementation. Journal of Computational Physics, 144:45-58, 1994.

[2] T. Aboiyar, E.H. Georgoulis, and A. Iske. Adaptive ADER Methods Using Kernel-Based Polyharmonic Spline WENO Reconstruction. SIAM Journal on Scientific Computing, 32:3251-3277, 2010.

[3] D. Balsara. Total variation diminishing scheme for relativistic magneto-hydrodynamics. The Astrophysical Journal Supplement Series, 132:83-101, 2001.

[4] D. Balsara and C.W. Shu. Monotonicity preserving weighted essentially non-oscillatory schemes with increasingly high order of accuracy. Journal of Computational Physics, 160:405-452, 2000.

[5] D. Balsara and D. Spicer. Maintaining pressure positivity in magnetohydrodynamic simulations. Journal of Computational Physics, 148:133-148, 1999.

[6] D. Balsara and D. Spicer. A staggered mesh algorithm using high order godunov fluxes to ensure solenoidal magnetic fields in magnetohydrodynamic simulations. Journal of Computational Physics, 149:270-292, 1999.

[7] D.S. Balsara. Self-adjusting, positivity preserving high order schemes for hydrodynamics and magnetohydrodynamics. Journal of Computational Physics, 231:7504-7517, 2012.

[8] D.S. Balsara, C. Meyer, M. Dumbser, H. Du, and Z. Xu. Efficient implementation of ADER schemes for euler and magnetohydrodynamical flows on structured meshes speed comparisons with rungekutta methods. Journal of Computational Physics, 235:934 - 969, 2013.

[9] D.S. Balsara, T. Rumpf, M. Dumbser, and C.D. Munz. Efficient, high accuracy ADER-WENO schemes for hydrodynamics and divergence-free magnetohydrodynamics. Journal of Computational Physics, 228:24802516, 2009.

[10] T.J. Barth and P.O. Frederickson. Higher order solution of the Euler equations on unstructured grids using quadratic reconstruction. AIAA paper no. 90-0013, 28th Aerospace Sciences Meeting January 1990.

[11] T.J. Barth and D.C. Jespersen. The design and application of upwind schemes on unstructured meshes. AIAA Paper 89-0366, pages 1-12, 1989.

[12] M. Ben-Artzi and J. Falcovitz. A second-order godunov-type scheme for compressible fluid dynamics. Journal of Computational Physics, 55:1-32, 1984.

[13] David J. Benson. Momentum advection on a staggered mesh. Journal of Computational Physics, 100(1):143 $-162,1992$.

[14] Jay P Boris and David L Book. Flux-corrected transport. I. SHASTA, a fluid transport algorithm that works. Journal of Computational Physics, 11(1):38 - 69, 1973.

[15] Jay P. Boris and David L. Book. Flux-corrected transport. Journal of Computational Physics, 135(2):172 $-186,1997$.

[16] J. Casper and H.L. Atkins. A finite-volume high-order ENO scheme for two-dimensional hyperbolic systems. Journal of Computational Physics, 106:62-76, 1993. 
[17] C. C. Castro and E. F. Toro. Solvers for the high-order riemann problem for hyperbolic balance laws. Journal of Computational Physics, 227:2481-2513, 2008.

[18] S. Clain, S. Diot, and R. Loubère. A high-order finite volume method for systems of conservation lawsmultidimensional optimal order detection (mood). Journal of Computational Physics, 230(10):4028 - 4050, 2011.

[19] B. Cockburn, S. Hou, and C. W. Shu. The Runge-Kutta local projection discontinuous Galerkin finite element method for conservation laws IV: the multidimensional case. Mathematics of Computation, 54:545$581,1990$.

[20] B. Cockburn, G. E. Karniadakis, and C.W. Shu. Discontinuous Galerkin Methods. Lecture Notes in Computational Science and Engineering. Springer, 2000.

[21] B. Cockburn, S. Y. Lin, and C.W. Shu. TVB Runge-Kutta local projection discontinuous Galerkin finite element method for conservation laws III: one dimensional systems. Journal of Computational Physics, 84:90-113, 1989.

[22] B. Cockburn and C. W. Shu. TVB Runge-Kutta local projection discontinuous Galerkin finite element method for conservation laws II: general framework. Mathematics of Computation, 52:411-435, 1989.

[23] B. Cockburn and C. W. Shu. The Runge-Kutta local projection P1-Discontinuous Galerkin finite element method for scalar conservation laws. Mathematical Modelling and Numerical Analysis, 25:337-361, 1991.

[24] B. Cockburn and C. W. Shu. The Runge-Kutta discontinuous Galerkin method for conservation laws V: multidimensional systems. Journal of Computational Physics, 141:199-224, 1998.

[25] P. Colella and M.D. Sekora. A limiter for PPM that preserves accuracy at smooth extrema. Journal of Computational Physics, 227:7069-7076, 2008.

[26] R. B. Dahlburg and J. M. Picone. Evolution of the orszagtang vortex system in a compressible medium. I. initial average subsonic flow. Phys. Fluids B, 1:2153-2171, 1989.

[27] A. Dedner, F. Kemm, D. Kröner, C.-D. Munz, T. Schnitzer, and M. Wesenberg. Hyperbolic divergence cleaning for the MHD equations. Journal of Computational Physics, 175:645-673, 2002.

[28] Vivien Desveaux and Christophe Berthon. An entropic mood scheme for the euler equations. submitted, 2013.

[29] S. Diot, S. Clain, and R. Loubère. Improved detection criteria for the multi-dimensional optimal order detection (MOOD) on unstructured meshes with very high-order polynomials. Computers and Fluids, $64: 43-63,2012$.

[30] S. Diot, R. Loubère, and S. Clain. The MOOD method in the three-dimensional case: Very-high-order finite volume method for hyperbolic systems. International Journal of Numerical Methods in Fluids, 73:362-392, 2013.

[31] M. Dubiner. Spectral methods on triangles and other domains. Journal of Scientific Computing, 6:345-390, 1991.

[32] M. Dumbser. Arbitrary high order PNPM schemes on unstructured meshes for the compressible NavierStokes equations. Computers \& Fluids, 39:60-76, 2010.

[33] M. Dumbser, D. Balsara, E.F. Toro, and C.D. Munz. A unified framework for the construction of onestep finite-volume and discontinuous Galerkin schemes. Journal of Computational Physics, 227:8209-8253, 2008.

[34] M. Dumbser, D. Balsara, E.F. Toro, and C.D. Munz. A unified framework for the construction of onestep finite volume and discontinuous galerkin schemes on unstructured meshes. Journal of Computational Physics, 227:8209-8253, 2008.

[35] M. Dumbser, M. Castro, C. Parés, and E.F. Toro. ADER schemes on unstructured meshes for nonconservative hyperbolic systems: Applications to geophysical flows. Computers and Fluids, 38:1731-1748, 2009.

[36] M. Dumbser, C. Enaux, and E.F. Toro. Finite volume schemes of very high order of accuracy for stiff hyperbolic balance laws. Journal of Computational Physics, 227:3971-4001, 2008.

[37] M. Dumbser and M. Käser. Arbitrary high order non-oscillatory finite volume schemes on unstructured meshes for linear hyperbolic systems. Journal of Computational Physics, 221:693-723, 2007.

[38] M. Dumbser, M. Käser, V.A Titarev, and E.F. Toro. Quadrature-free non-oscillatory finite volume schemes on unstructured meshes for nonlinear hyperbolic systems. Journal of Computational Physics, 226:204-243, 2007. 
[39] M. Dumbser and C.D. Munz. ADER discontinuous Galerkin schemes for aeroacoustics. Comptes Rendus Mécanique, 333:683-687, 2005.

[40] M. Dumbser and C.D. Munz. Building blocks for arbitrary high order discontinuous Galerkin schemes. Journal of Scientific Computing, 27:215-230, 2006.

[41] M. Dumbser and E. F. Toro. On universal Osher-type schemes for general nonlinear hyperbolic conservation laws. Communications in Computational Physics, 10:635-671, 2011.

[42] M. Dumbser and E. F. Toro. A simple extension of the Osher Riemann solver to non-conservative hyperbolic systems. Journal of Scientific Computing, 48:70-88, 2011.

[43] M. Dumbser and O. Zanotti. Very high order PNPM schemes on unstructured meshes for the resistive relativistic MHD equations. Journal of Computational Physics, 228:6991-7006, 2009.

[44] R.W. Dyson. Technique for very high order nonlinear simulation and validation. Technical Report TM2001-210985, NASA, 2001.

[45] B. Einfeldt, C. D. Munz, P. L. Roe, and B. Sjögreen. On godunov-type methods near low densities. Journal of Computational Physics, 92:273-295, 1991.

[46] G. Gassner, M. Dumbser, F. Hindenlang, and C.D. Munz. Explicit one-step time discretizations for discontinuous Galerkin and finite volume schemes based on local predictors. Journal of Computational Physics, 230:4232-4247, 2011.

[47] B. Giacomazzo and L. Rezzolla. The exact solution of the Riemann problem in relativistic magnetohydrodynamics. Journal of Fluid Mechanics, 562:223-259, 2006.

[48] S. Gottlieb and C.W. Shu. Total variation diminishing Runge-Kutta schemes. Mathematics of Computation, 67:73-85, 1998.

[49] A. Harten, B. Engquist, S. Osher, and S. Chakravarthy. Uniformly high order essentially non-oscillatory schemes, III. Journal of Computational Physics, 71:231-303, 1987.

[50] A. Harten, B. Engquist, S. Osher, and S.R. Chakravarthy. Uniformly high order accurate essentially non-oscillatory schemes III. Journal of Computational Physics, 71:231-303, 1987.

[51] A. Harten, P.D. Lax, and B. van Leer. On upstream differencing and godunov-type schemes for hyperbolic conservation laws. SIAM Review, 25(1):35-61, 1983.

[52] A. Harten and S. Osher. Uniformly highorder accurate nonoscillatory schemes i. SIAM J. Num. Anal., 24:279-309, 1987.

[53] A. Hidalgo and M. Dumbser. ADER schemes for nonlinear systems of stiff advection-diffusion-reaction equations. Journal of Scientific Computing, 48:173-189, 2011.

[54] V. Honkkila and P. Janhunen. HLLC solver for ideal relativistic MHD. Journal of Computational Physics, 223:643-656, 2007.

[55] C. Hu and C.W. Shu. Weighted essentially non-oscillatory schemes on triangular meshes. Journal of Computational Physics, 150:97-127, 1999.

[56] X.Y. Hu, N.A. Adams, and C.W. Shu. Positivity-preserving method for high-order conservative schemes solving compressible Euler equations. Journal of Computational Physics, 242:169-180, 2013.

[57] G.-S. Jiang and C.W. Shu. Efficient implementation of weighted ENO schemes. Journal of Computational Physics, 126:202-228, 1996.

[58] G.S. Jiang and C.C. Wu. A high-order WENO finite difference scheme for the equations of ideal magnetohydrodynamics. Journal of Computational Physics, 150:561-594, 1999.

[59] M. Käser and A. Iske. ADER schemes on adaptive triangular meshes for scalar conservation laws. Journal of Computational Physics, 205:486-508, 2005.

[60] V. P. Kolgan. Application of the minimum-derivative principle in the construction of finite-difference schemes for numerical analysis of discontinuous solutions in gas dynamics. Transactions of the Central Aerohydrodynamics Institute, 3(6):68-77, 1972. in Russian.

[61] Raphaël Loubère and Mikhail J. Shashkov. A subcell remapping method on staggered polygonal grids for arbitrary-lagrangian-eulerian methods. J. Comput. Phys., 209:105-138, 2005.

[62] H. Luo, L. Luo, R. Nourgaliev, V.A. Mousseau, and N. Dinh. A reconstructed discontinuous Galerkin method for the compressible NavierStokes equations on arbitrary grids. Journal of Computational Physics, 229:6961-6978, 2010.

[63] H. Luo, Y. Xia, S. Spiegel, R. Nourgaliev, and Z. Jiang. A reconstructed discontinuous Galerkin method 
based on a Hierarchical WENO reconstruction for compressible flows on tetrahedral grids . Journal of Computational Physics, 236:477-492, 2013.

[64] R.C. Millington, E.F. Toro, and L.A.M. Nejad. Arbitrary High Order Methods for Conservation Laws I: The One Dimensional Scalar Case. PhD thesis, Manchester Metropolitan University, Department of Computing and Mathematics, June 1999.

[65] S. A. Orszag and C. M. Tang. Small-scale structure of two-dimensional magnetohydrodynamic turbulence. Journal of Fluid Mechanics, 90:129, 1979.

[66] C. Palenzuela, L. Lehner, O. Reula, and L. Rezzolla. Beyond ideal MHD: towards a more realistic modeling of relativistic astrophysical plasmas. Mon. Not. R. Astron. Soc., 394:1727-1740, 2009.

[67] J. M. Picone and R. B. Dahlburg. Evolution of the orszag-tang vortex system in a compressible medium. II. supersonic flow. Phys. Fluids B, 3:29-44, 1991.

[68] J. Qiu and C.W. Shu. Finite difference WENO schemes with Lax-Wendroff type time discretization. SIAM J. Sci. Comput., 24(6):2185-2198, 2003.

[69] J. Qiu and C.W. Shu. Hermite WENO schemes and their application as limiters for Runge-Kutta discontinuous Galerkin method II: two dimensional case. Computers and Fluids, 34:642-663, 2005.

[70] J. Qiu and C.W. Shu. Runge-Kutta discontinuous Galerkin method using WENO limiters. SIAM Journal on Scientific Computing, 26:907-929, 2005.

[71] J. Reisner, J. Serencsa, and S. Shkoller. A spacetime smooth artificial viscosity method for nonlinear conservation laws. Journal of Computational Physics, 235(0):912 - 933, 2013.

[72] L. Rezzolla and O. Zanotti. An improved exact riemann solver for relativistic hydrodynamics. Journal of Fluid Mechanics, 449:395-411, 2001.

[73] V. V. Rusanov. Calculation of Interaction of Non-Steady Shock Waves with Obstacles. J. Comput. Math. Phys. USSR, 1:267-279, 1961.

[74] T. Schwartzkopff, M. Dumbser, and C.D. Munz. Fast high order ADER schemes for linear hyperbolic equations. Journal of Computational Physics, 197:532-539, 2004.

[75] T. Schwartzkopff, C.D. Munz, and E.F. Toro. ADER: A high order approach for linear hyperbolic systems in 2d. Journal of Scientific Computing, 17(1-4):231-240, 2002.

[76] J. Shi, C. Hu, and C.W. Shu. A technique of treating negative weights in WENO schemes. Journal of Computational Physics, 175:108-127, 2002.

[77] C.W. Shu. Essentially non-oscillatory and weighted essentially non-oscillatory schemes for hyperbolic Conservation Laws. NASA/CR-97-206253 ICASE Report No.97-65, November 1997.

[78] C.W. Shu and S. Osher. Efficient implementation of essentially non-oscillatory shock capturing schemes. Journal of Computational Physics, 77:439-471, 1988.

[79] A.H. Stroud. Approximate Calculation of Multiple Integrals. Prentice-Hall Inc., Englewood Cliffs, New Jersey, 1971.

[80] A. Suresh and H.T. Huynh. Accurate monotonicity-preserving schemes with runge-kutta time stepping. Journal of Computational Physics, 136:83-99, 1997.

[81] Huazhong Tang and Tiegang Liu. A note on the conservative schemes for the euler equations. Journal of Computational Physics, 218(2):451 - 459, 2006.

[82] A. Taube, M. Dumbser, D. Balsara, and C.D. Munz. Arbitrary high order discontinuous Galerkin schemes for the magnetohydrodynamic equations. Journal of Scientific Computing, 30:441-464, 2007.

[83] V.A. Titarev and E.F. Toro. ADER: Arbitrary high order Godunov approach. Journal of Scientific Computing, 17(1-4):609-618, December 2002.

[84] V.A. Titarev and E.F. Toro. ADER schemes for three-dimensional nonlinear hyperbolic systems. Journal of Computational Physics, 204:715-736, 2005.

[85] V.A. Titarev, P. Tsoutsanis, and D. Drikakis. WENO schemes for mixed-element unstructured meshes. Communications in Computational Physics, 8:585-609, 2010.

[86] E. F. Toro and V. A. Titarev. Derivative Riemann solvers for systems of conservation laws and ADER methods. Journal of Computational Physics, 212(1):150-165, 2006.

[87] E.F. Toro. Riemann Solvers and Numerical Methods for Fluid Dynamics. Springer, second edition, 1999.

[88] E.F. Toro and A. Hidalgo. ADER finite volume schemes for nonlinear reaction-diffusion equations . Applied Numerical Mathematics, 59:73-100, 2009. 
[89] E.F. Toro, R.C. Millington, and L.A.M Nejad. Towards very high order Godunov schemes. In E.F. Toro, editor, Godunov Methods. Theory and Applications, pages 905-938. Kluwer/Plenum Academic Publishers, 2001.

[90] E.F. Toro and V. A. Titarev. Solution of the generalized Riemann problem for advection-reaction equations. Proc. Roy. Soc. London, pages 271-281, 2002.

[91] E.F. Toro and V.A. Titarev. ADER schemes for scalar hyperbolic conservation laws with source terms in three space dimensions. Journal of Computational Physics, 202:196-215, 2005.

[92] P. Tsoutsanis, V.A. Titarev, and D. Drikakis. WENO schemes on arbitrary mixed-element unstructured meshes in three space dimensions. Journal of Computational Physics, 230:1585-1601, 2011.

[93] B. van Leer. Towards the ultimate conservative difference scheme II: Monotonicity and conservation combined in a second order scheme. Journal of Computational Physics, 14:361-370, 1974.

[94] B. van Leer. Towards the ultimate conservative difference scheme V: A second order sequel to Godunov's method. Journal of Computational Physics, 32:101-136, 1979.

[95] P. Woodward and P. Colella. The numerical simulation of two-dimensional fluid flow with strong shocks. Journal of Computational Physics, 54:115-173, 1984.

[96] L. Del Zanna, N. Bucciantini, and P. Londrillo. An efficient shock-capturing central-type scheme for multidimensional relativistic flows II. magnetohydrodynamics. Astronomy and Astrophysics, 400:397-413, 2003.

[97] L. Del Zanna, O. Zanotti, N. Bucciantini, and P. Londrillo. ECHO: an Eulerian conservative high order scheme for general relativistic magnetohydrodynamics and magnetodynamics. Astronomy and Astrophysics, 473:11-30, 2007.

[98] X. Zhang and C.W. Shu. Positivity-preserving high order finite difference WENO schemes for compressible Euler equations . Journal of Computational Physics, 231:2245-2258, 2012. 